Published in final edited form as:

Nature. 2016 March 17; 531(7594): 323-328. doi:10.1038/nature17181.

\title{
Lens regeneration using endogenous stem cells with gain of visual function
}

\author{
Haotian Lin ${ }^{1,{ }^{*}}$, Hong Ouyang ${ }^{1,{ }^{*}}$, Jie Zhu ${ }^{2,{ }^{*}}$, Shan Huang ${ }^{1,{ }^{*},}$ Zhenzhen Liu ${ }^{1}$, Shuyi Chen ${ }^{1}$, \\ Guiqun $\mathrm{Cao}^{3}$, Gen $\mathrm{Li}^{3,4}$, Robert A. J. Signer ${ }^{5}$, Yanxin $\mathrm{Xu}^{3,6}$, Christopher Chung ${ }^{2}$, Ying \\ Zhang ${ }^{7}$, Danni Lin², Sherrina Patel ${ }^{2}$, Frances $\mathrm{Wu}^{2}$, Huimin Cai ${ }^{3,4}$, Jiayi Hou ${ }^{8}$, Cindy Wen², \\ Maryam Jafari ${ }^{2}$, Xialin Liu ${ }^{1}$, Lixia Luo ${ }^{1}$, Jin Zhu ${ }^{2}$, Austin Qiu², Rui Hou ${ }^{4}$, Baoxin Chen ${ }^{1}$, \\ Jiangna Chen ${ }^{1}$, David Granet ${ }^{2}$, Christopher Heichel ${ }^{2}$, Fu Shang ${ }^{1}$, Xuri Li ${ }^{1}$, Michal \\ Krawczyk $^{2}$, Dorota Skowronska-Krawczyk ${ }^{2}$, Yujuan Wang ${ }^{1}$, William Shi ${ }^{2}$, Daniel Chen ${ }^{2}$, \\ Zheng Zhong ${ }^{1,2}$, Sheng Zhong ${ }^{2}$, Liangfang Zhang ${ }^{2}$, Shaochen Chen $^{2}$, Sean J. Morrison ${ }^{5}$, \\ Richard L. Maas ${ }^{7}$, Kang Zhang ${ }^{1,2,3,9}$, and Yizhi Liu ${ }^{1}$ \\ ${ }^{1}$ State Key Laboratory of Ophthalmology, Zhongshan Ophthalmic Center, Sun Yat-sen University, \\ Guangzhou 510060, China \\ ${ }^{2}$ Shiley Eye Institute, Institute for Engineering in Medicine, Institute for Genomic Medicine, \\ University of California, San Diego, La Jolla, California 92093, USA \\ ${ }^{3}$ Molecular Medicine Research Center, State Key Laboratory of Biotherapy, West China Hospital, \\ Sichuan University, Sichuan 610041, China \\ ${ }^{4}$ Guangzhou KangRui Biological Pharmaceutical Technology Company, Guangzhou 510005, \\ China \\ ${ }^{5}$ Howard Hughes Medical Institute, Children's Research Institute, Department of Pediatrics, \\ University of Texas Southwestern Medical Center, Dallas, Texas 75390, USA \\ ${ }^{6}$ Department of Ophthalmology, West China Hospital, Sichuan University, Sichuan 610041, China \\ ${ }^{7}$ Division of Genetics, Department of Medicine, Brigham and Women's Hospital and Harvard \\ Medical School, Boston, Massachusetts 02115, USA \\ ${ }^{8}$ Clinical and Translational Research Institute, University of California, San Diego, La Jolla, \\ California 92093, USA
}

\footnotetext{
Reprints and permissions information is available at www.nature.com/reprints.

Correspondence and requests for materials should be addressed to Y.L. (yzliu62@yahoo.com) or K.Z. (kang.zhang@gmail.com). These authors contributed equally to this work.

Online Content Methods, along with any additional Extended Data display Items and Source Data, are available in the online version of the paper; references unique to these sections appear only in the online paper.

Supplementary Information is available in the online version of the paper.

Author Contributions

H.L., S.H., Z.L., S.C., .X.L., L.L., B.C., Y.W. and Y.L. conducted the clinical trial; H.O., Jie Z., Y.Z., J.C., H.C. and S.P performed mouse LEC lineage tracing experiments; H.O., J.Z., G.C., G.L., Y.X., S.P., Jin Z., M.J., A.Q., F.S., X.L., R.H., W.S. and D.C. performed LEC characterization and differentiation experiments; D.G., C.H., F.W., Z.S. and J.H. analysed clinical trial data; H.O., M.K., D.S-K., C.C., M.J., Y.W., W.S., D.C., S.Z., L.Z. and S.C. performed gene expression studies and analysed data; R.A.J.S. and S.J.M. performed and analysed the experiments related to BMI-1 function in mouse lens epithelium. Y.L, R.M. and K.Z. designed the study and wrote the paper. All authors discussed the results and commented on the manuscript.

The authors declare no competing financial interests. Readers are welcome to comment on the online version of the paper.
} 
${ }^{9}$ Veterans Administration Healthcare System, San Diego, California 92093, USA

\section{Abstract}

The repair and regeneration of tissues using endogenous stem cells represents an ultimate goal in regenerative medicine. To our knowledge, human lens regeneration has not yet been demonstrated. Currently, the only treatment for cataracts, the leading cause of blindness worldwide, is to extract the cataractous lens and implant an artificial intraocular lens. However, this procedure poses notable risks of complications. Here we isolate lens epithelial stem/progenitor cells (LECs) in mammals and show that Pax 6 and Bmil are required for LEC renewal. We design a surgical method of cataract removal that preserves endogenous LECs and achieves functional lens regeneration in rabbits and macaques, as well as in human infants with cataracts. Our method differs conceptually from current practice, as it preserves endogenous LECs and their natural environment maximally, and regenerates lenses with visual function. Our approach demonstrates a novel treatment strategy for cataracts and provides a new paradigm for tissue regeneration using endogenous stem cells.

Stem-cell therapy holds great promise in regenerative medicine. Much attention has been focused on pluripotent stem cells and the use of their derivatives for therapeutic purposes. However, several uncertainties, including tumorigenicity and immune rejection, have hindered their clinical application. An attractive alternative is to harness the potential of endogenous stem/progenitor cells for direct use in repair and regeneration. In the case of the ocular lens, regeneration has been reported in lower vertebrates ${ }^{1,2}$. In mammals, such as rabbits, removal of the original lens content results in the proliferation of residual lens epithelial stem/progenitor cells (LECs) and the generation of a limited amount of lens fibres $^{3,4}$. In humans, varying degrees of disorganized regrowth of doughnut-like lens tissues have been observed after congenital cataract removal in infants (Extended Data Fig. 1a, b). However, the underlying mechanism for these observations remains elusive, and the successful regeneration of a complete mammalian lens with biological function has yet to be achieved.

Cataracts are the leading cause of blindness in the world ${ }^{5}$. The visual axis, defined as the normal passage of light into the eye, may undergo visual axis opacification (VAO) owing to the cataractous lens or the postoperative disorganized growth of remaining LECs, leading to vision $\operatorname{loss}^{6}$. The current standard of care in congenital cataracts involves surgical removal of the cataractous lens with a large central capsulorhexis opening and implantation of an artificial intraocular lens (IOL) to replace the missing refractive media. Although artificial IOLs are widely used in paediatric cataract surgery, they are limited by complications ${ }^{7,8}$, and most paediatric patients continue to require some form of refractive correction such as eyeglasses after cataract surgery ${ }^{9}$. Furthermore, IOLs are controversial in patients younger than two years as they have not been shown to prevent strabismus or amblyopia, and normal lens refractive power is not yet fully developed at this age ${ }^{10,11}$.

To test the feasibility of in situ lens regeneration, we performed in vitro studies on $\mathrm{PAX}^{+} /$ SOX $2^{+}$LECs and identified BMI-1 as an essential factor for maintaining a LEC pool in mammalian eyes by conditional knockout experiments. We also investigated the ability of 
LECs to differentiate into lens fibre cells in vitro. We then performed in vivo animal studies by establishing a new minimally invasive capsulorhexis surgery method that differs conceptually from current practice in extracting the cataractous lens through a small wound opening, while preserving lens capsule integrity and therefore LECs as well. Using this method, we investigated lens regeneration in rabbits and macaques and conducted a clinical trial in human infants. Functional lens regeneration was observed not only in rabbits and macaques, but also in human patients with congenital cataracts. Therefore, our new study provides a novel approach to lens regeneration using endogenous stem cells, and results in improved outcomes.

\section{Essential role of LECs in lens regeneration}

In the mature lens, LECs cover the anterior surface of the lens and begin to differentiate into lens fibres at the equator (Extended Data Fig. 2a). Sustained self-renewal and protective capacities against external injury and oxidative damage are among the most significant functions of LECs ${ }^{12}$. To assess the regenerative ability of LECs, we used bromodeoxyuridine (BrdU) labelling to identify proliferating LECs from human donor lenses. We quantified BrdU ${ }^{+}$LECs in 8-month-old, 30-year-old, and 40-year-old donors and found that the number of proliferating cells decreased with age (Extended Data Fig. 2b, c). However, upon surgical removal of the entire lens contents with preservation of the empty capsular bag scaffold, the number of $\mathrm{BrdU}^{+}$cells increased by 11 -fold $(\mathrm{P}<0.05$, Extended Data Fig. 2d, e), suggesting a strong regenerative capacity of human LECs after injury.

Pax6 plays a central role in eye development as well as in lens induction. After birth, Pax6 maintains a high level of expression in the lens epithelium, particularly at the germinative zone (Fig. 1a). To determine whether Pax6 $6^{+}$LECs can contribute to lens fibre cell formation, we performed lineage-tracing experiments in mice by crossing a Pax 6 lens ectoderm enhancer-driven Cre deleter mouse strain (P0-3.9-GFPcre) with the $R O S A^{m T m G}$ membranebound GFP reporter strain. We observed intense membrane $\mathrm{GFP}^{+}$cells throughout the entire lens of ROSA ${ }^{m T m G}$; Pax6P0-3.9-GFPcre mice at P1, P14, and P30; in contrast, the P0-3.9GFPCre allele alone yielded only nuclear GFP expression in LECs detectable by anti-GFP antibody staining (Fig. 1a, b). These results indicate that Pax6 $6^{+}$LECs from the embryonic or adult lens can contribute to the post-natal replacement of mouse lens fibre cells.

We next isolated and expanded rabbit LECs from neonatal lens capsules (see Methods). These LECs showed a cobble-stone-like epithelial morphology with highly positive staining for LECs markers Pax6 and Sox2, and could be passaged over time (Fig. 2a). Upon differentiation, these LECs formed transparent three-dimensional convex lens-like structures, defined as lentoid bodies (Fig. 2b, c), which possess significant refractive power (Fig. 2c). Immunostaining and western blot analysis showed that lentoid bodies expressed mature lens-fibre-specific genes, including those encoding aA-, $\beta$-, and $\gamma$-crystallins (Fig. $2 b, c)$. 


\section{Loss of LEC homeostasis leads to cataracts}

To further investigate the LEC pool and its role in the maintenance of lens function, we studied BMI-1, a member of the Polycomb-group family. BMI-1 is known to promote the maintenance and self-renewal of stem cells in multiple post-natal tissues ${ }^{13-15}$ and is expressed in both the murine lens germinative zone and in cultured human fetal LECs (Extended Data Fig. 2f, g and 3a). Knockdown of BMI1 in human LECs led to significantly decreased LECs proliferation in vitro (Extended Data Fig. 4a), without affecting expression of key genes in LECs or lens fibre cells (Extended Data Fig. 4b). To directly test the effects of conditional deletion of Bmil on LEC proliferation, we administered BrdU to 2-, 7-, and 12-month-old Nestin-cre;Bmil $1^{f l / f l}$ mice and Bmi $1^{f l / f l}$ littermate controls. After a 20-h pulse, there was no significant difference in the percentage of BrdU ${ }^{+}$LECs in 2-month-old Nestincre; $B m i 1^{f l / f l}$ mice and $B m i 1^{f l / f l}$ controls. However, there was a significant reduction in the percentage of $\mathrm{BrdU}^{+}$LECs in 7- and 12-month-old Nestin-cre;Bmi $1^{f l / f l}$ eyes compared to controls (Extended Data Fig. 3b, $P<0.05$ ).

We next investigated the mRNA expression levels of Bmil, Sox 2 and Ki67 in Pax6 ${ }^{+}$LECs at the anterior capsule in Pax6P0-3.9-GFPcre mouse lens. Compared with Pax6 ${ }^{-}$(GFPnegative) LECs, Pax6 ${ }^{+}$(GFP-positive) LECs located at the germinative zone had higher expression levels of Bmil, Sox 2 and Ki67 (Extended Data Fig. 5). Moreover, conditional deletion of $B m i 1$ led to a dramatic decrease in the number of Pax $6^{+} /$Sox $2^{+}$LECs in ageing Nestin-cre;Bmi $1^{\text {fl/fl }}$ mice (Extended Data Fig. 3a, $P<0.001$ ). Additionally, the lenses of ageing Nestin-cre;Bmi1 ${ }^{\mathrm{fl} / \mathrm{fl}}$ mice became progressively opaque, suggesting cataract formation.

To test this hypothesis, we administered tropicamide drops to the eyes of 2-, 7-, and 12month-old Nestin-cre;Bmi $1^{f l / f l}$ mice and Bmi ${ }^{f l / f l}$ littermate controls to dilate the pupils (Extended Data Fig. 3c, d). Eyes of 2-month-old Nestin-cre;Bmi1 $1^{f l / f l}$ mice $(n=3)$ were indistinguishable from those of age-matched controls $(n=4)$. However, $100 \%$ of the 7month-old $(n=5)$ and 12-month-old $(n=7)$ Nestin-cre; Bmi1 $1^{f l / f l}$ mice had bilateral cataracts, while none of the age-matched Bmi1 ${ }^{f l / f l}$ controls ( $n=3$, 7-month-old; $n=5$, 12-month-old) developed cataracts. Moreover, haematoxylin and eosin-stained sections revealed the presence of cataracts in the 7- and 12-month-old Nestin-cre;Bmi1 $1^{f l / f l}$ mice (Extended Data Fig. 3d). These data suggest that Bmil loss of function disrupted LEC proliferation, thereby depleting the LEC pool and promoting cataract formation.

\section{LEC preservation and lens regeneration}

The current capsulorhexis method performed in paediatric cataract surgery involves making a large 6-mm-diameter opening at the centre of the anterior capsule, resulting in a large wound area and destruction of large numbers of LECs (Extended Data Fig. 1c). To overcome these limitations and to facilitate lens regeneration, we established a new capsulorhexis method. This new method has two advantages: (1) it reduces the size of the wound considerably; and (2) it moves the capsulorhexis opening from the central visual axis to the periphery. Thus, application of this procedure led to improved visual axis transparency and preservation of LECs with regenerative potential (Fig. 3a). 
We next investigated lens regeneration in rabbit eyes in vivo. We used our new minimally invasive capsulorhexis technique to preserve endogenous LECs while removing the native lens (Extended Data Fig. 6). One day after surgery, slit-lamp microscopy showed that the anterior and posterior capsules were adherent (Fig. 3b). Four to five weeks after surgery, the regenerating lens tissue grew from the periphery of the capsular bag towards the centre in a curvilinear symmetrical pattern (Fig. 3b). Seven weeks after surgery, the regenerating lens tissue formed a transparent biconvex lens along the anterior-posterior axis with a clear view of the posterior segment and retina (Fig. 3b, c), comparable to a normal healthy lens (Fig. $3 c)$. The refractive power of the regenerated lenses after surgery was evaluated and found to have increased to an average of 15.6 dioptres from the first to the fifth month after surgery, a value comparable to that of a normal lens ${ }^{16}$ (Fig. 3d, $P<0.01$ ).

In contrast to the epithelial cells and premature lens fibre cells located at the lens equator, the LECs in the germinative zone of regenerated lenses showed intense proliferative activity 7 weeks post-surgery, as evidenced by both Ki67 and BrdU labelling (Fig. 3e-g). Notably, some PAX6 ${ }^{+}$LECs co-labelled with BrdU, demonstrating their proliferative potential (Fig. $3 \mathrm{~g})$. These LECs lost PAX6 expression concomitant with the initiation of differentiation and subsequent migration from the lens equator.

One day post-surgery, histological examination revealed that a monolayer of LECs remained intact (Extended Data Fig. 7a). Four days post-surgery, LECs migrated onto the posterior capsule from the periphery towards the centre in a curvilinear $360^{\circ}$ fashion with a single layer of epithelium on the posterior capsule (Extended Data Fig. 7a). Seven days postsurgery, LECs on the posterior capsule began to elongate, and their nuclei were positioned anteriorly away from the posterior capsule (Extended Data Fig. 7a). Twenty-eight days postsurgery, a structure with lens fibres and an extruded nucleus was observed (Extended Data Fig. 7b). At week 7 after surgery, the regenerated lens fibres elongated along the anteriorposterior axis and grew to cover the entire posterior capsular area, forming a lens with a double-convex shape (Extended Data Fig. 7c).

We next investigated lens regeneration in macaques 1-3 months of age (approximately equivalent to human infants 4-12 months old), using a similar minimally invasive surgical technique. From postoperative days 1 to 3 , no signs of inflammation or other undesired sideeffects were seen. Two to three months post-surgery, regenerating lens tissue had grown from the periphery towards the centre in a curvilinear pattern (Fig. 4a). Five months postsurgery, a biconvex lens with a transparent visual axis had formed (Fig. 4a, b). Fundus examination 7 weeks after surgery showed a clear view of the retina, comparable to the view of the retina seen through a normal healthy lens. No undesired complications, such as macular oedema, retinal detachment, or endophthalmitis were observed.

\section{Lens regeneration in human infants}

Cataracts are a major cause of vision loss in human infants ${ }^{17}$. Currently, the most commonly practiced surgical procedure involves removal of the cloudy lens through a large anterior continuous curvilinear capsulorhexis (ACCC), combined with either posterior laser capsulotomy or posterior continuous curvilinear capsulorhexis (PCCC) and anterior 
vitrectomy (Extended Data Fig. 1), which is followed by artificial lens implantation or postoperative aphakic eyeglasses or contact lenses ${ }^{18}$. However, complications such as VAO often occur. Moreover, difficulty with refractive correction of developing eyes, secondary glaucoma, and surgery-related complications can lead to a poor outcome ${ }^{19}$. We conducted a clinical trial in paediatric cataract patients up to two years of age to investigate whether lenses could be regenerated in humans using minimally invasive surgery.

Twelve paediatric cataract patients ( 24 eyes) underwent minimally invasive surgery to promote lens regeneration, while 25 paediatric cataract patients (50 eyes) in the control group received the current standard-of-care treatment that left them aphakic. Using slit-lamp microscopy, we were able to dynamically observe the process of in vivo lens regeneration postoperatively. The capsular openings healed within one month after minimally invasive surgery. Three months postsurgery, a regenerated transparent biconvex lens structure had formed (Extended Data Fig. 8c, d). No significant VAO or other complications were observed at 6 months post-surgery (Table 1).

All of the eyes gained visual function when the capsular bag was refilled with a regenerated lens of relatively uniform density. A clear view of the fundus was observed in all cases with successful lens regeneration (Extended Data Fig. 8c, d). The average central thickness of the regenerated lenses increased significantly after surgery and was comparable to a native lens at 8 months post-surgery (Fig. 5a, $P<0.01$ ). We also used retinoscopy and ophthalmoscopy to evaluate the function of the regenerated lenses and found that from the first week to 8 months post-surgery, the refractive power increased significantly (Fig. 5b, $n=24, P<0.01$ ).

The accommodative ability of the regenerated lenses was evaluated 8 months after surgery using an open-field autorefractor (see Methods). The mean accommodative response increased to 2.5 dioptres in regenerated lenses, which was markedly improved compared to the 0.10 dioptre increase in aphakic controls $(\mathrm{P}<0.001)$. Grating acuities (cycles/degree) was recorded preoperatively and at each postoperative follow-up appointment, then converted to the logarithm of the minimum angle of resolution. Infant visual acuity and accommodation power were significantly improved postoperatively compared to the preoperative baseline $(\mathrm{P}<0.001)$ (Fig. $5 \mathrm{c}, \mathrm{d})$. The increase in visual acuity was comparable to that achieved using the current surgical method (Fig. 5e). Thus, visual function testing showed that the regenerated lenses were functional.

\section{Clinical outcome with new or current treatment approach}

It is well known that with the current method for paediatric cataract surgery, VAO will occur in nearly all patients weeks or months postoperatively owing to the abnormal proliferation of residual LECs $^{20}$ (Table 1). To avoid VAO, additional procedures such as polishing of the lens capsule, laser capsulotomy, PCCC, and anterior vitrectomy are widely practiced to disrupt LECs, the lens capsule on which LECs proliferate, and aberrant lens fibre regeneration. Although these procedures can decrease VAO incidence by $15 \%$, they carry significant risk of postoperative inflammation and complications. In this clinical trial, our minimally invasive surgical method resulted in visual axis transparency in nearly all eyes (95.8\%) (Fig. 5f, Table 1). Since the scar from the ACCC was $<1.5 \mathrm{~mm}$ in diameter and 
located in the periphery of the anterior capsule, it was far from the visual axis (Fig. 5f) and not visible unless the pupils were dilated. The preserved lens capsule remained nearly entirely transparent (Fig. 5f). No disorganized tissue regeneration was observed. Thus, compared to the current standard of care for cataract surgery, our new minimally invasive technique decreased VAO by more than 20 -fold ( $84 \%$ versus $4.2 \%$ ). Furthermore, there was an intact posterior capsule and lens-vitreous interface (Table 1).

By using paired t-tests within each group, significant improvement of decimal acuity before and after treatment was observed with $P<0.001(t=23.40$, degrees of freedom (d.f.) $=$ 49.04) in the standard-of-care group and $P<0.001(t=15.05$, d.f. $=23.01)$ in the novel treatment group. A linear mixed-effect model using decimal acuity as the outcome (time: baseline, 1 week, 3 month (after surgery for control group)) and treatment assignment and their interaction as fixed effects yielded statistically insignificant result for time and treatment interaction by likelihood ratio test with $P=0.956\left(X^{2}=0.332\right.$, d.f. $\left.=3\right)$ (Extended Data Table 1a, left, and Extended Data Table 1c, left)), which indicated that the mean response profiles for the two groups were parallel over time. We then refit the linear mixedeffect model by dropping out the interaction term (Extended Data Table 1b, left). A likelihood ratio test with insignificant $P$ value of $0.776\left(X^{2}=0.081\right.$, d.f. $\left.=1\right)$ (Extended Data Table 1c, left), suggested that the difference between mean decimal acuity in two groups was not statistically different over time (Extended Data Fig. 8b). In contrast, the linear mixedeffect model using decimal acuity as the outcome (time: baseline, 1 week, 3 month (before surgery for control group)) and treatment assignment and their interaction as fixed effects yielded statistically significant results for time and treatment interaction with $P<0.001\left(X^{2}=\right.$ 47.529, d.f. =3) (Extended Data Table 1a, right, and Extended Data Table 1c, right). The non-parallel pattern of mean responses from two groups was largely due to vision loss at 3 months before laser surgery in the control group, while the decimal acuity was monotonically increased in the novel treatment group (Extended Data Fig. 8b). The novel treatment also shows significantly lower complication rate by almost every measurement, supporting the superiority and safety of the novel treatment (Table 1).

\section{Discussion}

Each year, more than 20 million cataract patients worldwide undergo treatment with lens extraction and artificial IOL implantation ${ }^{5}$. Despite the clinical success of IOLs, they have numerous limitations and potential complications, including IOL dislocation, suboptimal biocompatibility, inadequate accommodation, and poor visual outcomes. In the worst cases, irreversible blindness may result ${ }^{21}$. Thus, a new strategy for treating congenital cataracts using naturally regenerated lenses is highly desirable.

The current surgical procedure for paediatric cataracts impairs lens regeneration in several ways. First, the commonly used ACCC creates a relatively large opening at the centre of the anterior capsule, prolonging recovery time and increasing the incidence of inflammation, while wound healing may form scars and cause postoperative VAO. Second, the surgical procedure removes most of the anterior subcapsular LECs, of which a subpopulation is critical for lens regeneration ${ }^{22}$. Third, abnormal proliferation of residual LECs causes postoperative VAO in many cases ${ }^{23}$, which requires opening of the posterior capsule, 
performed by either laser capsulotomy or PCCC and anterior vitrectomy ${ }^{24}$. By destroying the integrity of the lens capsule and LECs, the current surgical procedure greatly diminishes the possibility of lens regeneration ${ }^{25}$.

In this study, we show that a new minimally invasive cataract surgery method preserves the integrity of the lens capsule and associated LECs, facilitating functional lens regeneration in animals and humans. In addition to achieving lens regeneration in patients with congenital cataracts, our method also increased visual axis transparency and decreased the rate of complications. The small capsulorhexis opening healed quickly with a nearly intact lens capsule and minimal postoperative inflammation (Fig. 5f, Extended Data Fig. 8c-e). After surgery, there was a clear cornea, anterior chamber, and fundus, with no surgery-related complications.

Our method resulted in visual axis transparency in $>95 \%$ of cataractous eyes in infants, a much higher percentage than that obtained by traditional surgery. In the remaining patient with VAO, some degree of opacification of the regenerated lens occurred that correlated with improper healing of the anterior capsulorhexis opening and loss of LECs. Therefore, visual axis transparency necessitates preservation of the integrity of the lens capsule and associated LECs, as was illustrated by our lens regeneration study in rabbits. Furthermore, we found that BMI-1 is required for maintenance and renewal of endogenous LECs, and that loss of BMI-1 leads to reduction of the proliferative capacity of LECs and cataract formation. The fact that LECs in adult human eyes exhibit increased proliferative potential after injury highlights the potential for LEC replenishment beyond the paediatric population. These findings may therefore have implications for lens regeneration in elderly patients with agerelated cataracts. However, there are important differences between paediatric and adult cataracts. Hard cataracts (nuclear sclerosis) in adults may require phacoemulsification, which could damage LECs; in addition, tissue consistency and capsular thickness/elasticity may pose other challenges for adult lens regeneration. Furthermore, differences between the regenerative capacities of paediatric and adult lenses may suggest a prolonged period of regeneration in adults.

In summary, the current surgical procedure for cataract treatment inadvertently destroys the integrity of the lens capsule and the very LECs that hold the regenerative key to lens restoration. It is also associated with numerous side-effects and a significant risk of complications, particularly in infants. To overcome these problems, we have developed a new, minimally invasive surgical method that allows regeneration of a functional lens with refractive and accommodative abilities, and with greater visual axis transparency. This new cataract treatment uses endogenous stem cells to replenish the human ocular lens, and provides a fresh paradigm for organ and tissue regeneration.

\section{METHODS}

\section{Isolation and culture of LECs}

All animal studies were performed with the approval of the Institutional Animal Care Committees of Sun Yat-sen University, the University of California San Diego, West China Hospital, and the University of Texas Southwestern Medical Center. 
The eyeball was enucleated from a one-month-old New Zealand white rabbit and washed with PBS (containing antibiotics) three times. After the cornea and iris were removed, a small cut was made in the posterior capsule of the lens; the capsule with attached epithelium was removed and cut into $1 \times 1 \mathrm{~mm}^{2}$ pieces. The pieces of epithelium were cultured in minimum essential media supplemented with $20 \%$ FBS, NEAA, and $50 \mu \mathrm{g} \mathrm{ml}^{-1}$ gentamicin.

A 17-week-old human fetal eyeball was purchased from Advanced Bioscience Resources, Inc. (San Francisco, California). Post-mortem human eyes were obtained from San Diego Eye Bank. The human LECs were cultured according to the same methods as above.

For in vitro differentiation, LECs were cultured on Matrigel-coated six-well plates or eightwell chambers. Lentoid body was formed after 21 days in minimum essential media supplemented with NEAA, $1 \% \mathrm{FBS}, 100 \mathrm{ng} \mathrm{ml}^{-1} \mathrm{FGF} 2$, and $5 \mu \mathrm{g} \mathrm{ml}^{-1}$ insulin. Images of lentoid tissue were obtained using a Leica M205FA stereo microscope.

\section{Transgenic mouse study}

Membrane-tomato/membrane-green (mTmG)-targeted $R O S A^{m T m G}$ mice were purchased from the Jackson Laboratory (Bar Harbour, ME; stock no. 7576) and maintained as homozygotes. P0-3.9-GFPcre mice expressing an eGFP-Cre recombinase fusion protein under the control of the Pax 6 lens ectoderm enhancer and the Pax 6 PO promoter $^{26}$ were maintained in a FVB/N background. Lineage-tracing experiments were performed by crossing the homozygous $R O S A^{m T m G}$ reporter mouse strain with the P0-3.9-GFPcre deleter strain. Eyes were dissected at P1, P14, and P30 and fixed overnight in $4 \%$ formaldehyde. Tissues were then incubated in 10\% sucrose and embedded in OCT for cryo-sectioning. Frozen sections were washed in PBS and imaged on a Zeiss Axio Imager fluorescence

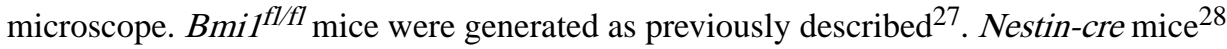
were obtained from the Jackson Laboratory. For BrdU pulses, mice were injected with 100 $\mathrm{mg} \mathrm{kg}^{-1} \mathrm{BrdU}$ (Sigma) dissolved in PBS, then maintained on drinking water that contained $1 \mathrm{mg} \mathrm{ml}^{-1}$ BrdU until sacrifice.

For gene expression studies, lenses of Pax6P0-3.9-GFPcre mice were dissected under a dissecting microscope. Lens capsular bag was opened from the posterior surface by making three crisscross incisions. The capsular bag was opens and lens material extruded. GFPpositive LECs in the mid-anterior capsular area were separated mechanically from GFPnegative LECs in the remaining capsular areas under a fluorescence microscope. RNA was isolated using RNeasy Mini Kit (Qiagen).

To image cataracts, mice were anaesthetized with Avertin, and one drop of 1\% Mydriacyl (Alcon) was administered per eye. Eyes were immediately visualized in vivo using a light microscope. For histology, mice were perfused with heparinized saline followed by $4 \%$ paraformaldehyde (PFA) in PBS. Dissected eyes were fixed in 4\% PFA overnight, embedded in paraffin, and sectioned by the UT Southwestern Molecular Pathology core facility. For BrdU staining, slides were deparaffinized, and subjected to heat-mediated antigen retrieval (in $10 \mathrm{mM}$ sodium citrate, $\mathrm{pH}$ 6.0). Slides were stained with primary mouse anti-BrdU (Caltag, MD5000, 1:200) overnight at $4{ }^{\circ} \mathrm{C}$. Slides were subsequently stained with Alexa Fluor 555-conjugated goat anti-mouse IgG1 secondary antibody (Life Technologies, 1:500) 
and $1 \mathrm{mg} \mathrm{ml}^{-1}$ DAPI (1:500) for $1 \mathrm{~h}$ at room temperature. The number of BrdU-labelled cells was divided by the total number of $\mathrm{DAPI}^{+}$cells in a single layer of LECs.

\section{Lentiviral RNAi}

Lentiviral shRNA targeting the human $B M I 1$ gene (NCBI Reference Sequence:

NM_005180.8) was purchased from Origene (TL314462), ShRNA targeting sequences were as follow: 5' -AATGCCATATTGGTATATGA CATAACAGG-3' and 5' -

GTAAGAATCAGATGGCATTATGCTTGTTG-3'. Two shRNAs were used separately, and a non-effective 29-mer scrambled shRNA was used as a control. Lentiviral shRNA particles were prepared using shRNA lentiviral packaging kit (Origene, TR30022). Viruses were harvested at $48 \mathrm{~h}$ and $72 \mathrm{~h}$ post-transfection.

\section{Western blot analysis}

LECs were cultured on Matrigel-coated 3.5-mm dishes with lentoid formation medium for 30 days. Cells were washed twice with ice-cold PBS, and lysed in RIPA lysis buffer with PMSF. Protein concentration was determined by BCA protein assay kit. Thirty micrograms of total protein lysate was loaded onto 10\% SDS-PAGE gel and then transferred to a PVDF membrane (Millipore) at $70 \mathrm{~V}$ for $2 \mathrm{~h}$. The membrane was probed with the following primary antibody at $4{ }^{\circ} \mathrm{C}$ overnight: anti-aA-crystallin (sc-22389, Santa Cruz), anti- $\beta$ crystallin (sc-48335, Santa Cruz), anti- $\gamma$-crystallin (sc-22415, Santa Cruz) and anti- $\beta$-actin (sc-47778, Santa Cruz), and then incubated with HRP-conjugated anti-rabbit, anti-mouse, or anti-goat secondary antibody for $1 \mathrm{~h}$ at room temperature. The immunodetection was visualized using a blot imaging system (Fluor Chem Q, Protein Simple) with ECL buffer (Millipore).

\section{Lens regeneration in rabbit and macaque models}

New Zealand white rabbits ( $n=29$, four rabbits died from systemic infections unrelated to surgery. The remaining 25 rabbits were used to assess regeneration), and long-tailed macaques (Macaca fascicularis) monkeys $(n=6)$ underwent minimally invasive capsulorhexis surgery. Only the left eye of each animal was used for experiments. Slit-lamp biomicroscopy and photography were performed at different time points to monitor lens regeneration. Rabbits were euthanized at day 1 , day 7 , and one month after surgery, and the treated eyes were enucleated. The lenses were harvested for histologic analysis using haematoxylin and eosin staining. For the macaques, enucleation of the treated eye was performed 4 months post-surgery and the lenses were harvested for the same histologic examinations. The eyes were fixed, paraffin-embedded, and sectioned at $5 \mu \mathrm{m}$ through the cornea, pupil, and optic nerve with the lens in situ.

\section{Real-time PCR}

RNA was isolated from rabbit LECs, mature lens fibre cells and LECs in P0-3.9-GFPcre mice using an RNeasy Mini Kit (Qiagen) and subjected to on-column DNase digestion. cDNA was synthesized using a Superscript III reverse transcriptase kit according to the manufacturer's instructions (Invitrogen). Quantitative PCR was performed via 40 cycle amplification using gene-specific primers (Supplementary Table 1) and Power SYBR Green 
PCR Master Mix on a 7500 Real-Time PCR System (Applied Biosystems). Measurements were performed in triplicate and normalized to endogenous GAPDH levels. The relative fold change in expression was calculated using the $\Delta \Delta C_{\mathrm{t}}$ method $\left(C_{\mathrm{t}}\right.$ values $\left.<30\right)$.

\section{Immunofluorescence and laser confocal microscopy}

Rabbit LECs were fixed in 4\% PFA for 20 min, then permeabilized with $0.3 \%$ Triton X-100PBS for 10 min and blocked in PBS solution containing 5\% BSA, followed by an overnight incubation in primary antibodies at $4{ }^{\circ} \mathrm{C}$. After three washes in PBS, cells were incubated with secondary antibody for $1 \mathrm{~h}$ in room temperature. Cell nuclei were counterstained with DAPI.

The following antibodies were used: goat anti-Sox2 polyclonal antibody (Santa Cruz), rabbit anti-PAX6 polyclonal antibody (PRB-278P, Covance), mouse anti-Bmi1 antibody (ab14389, Abcam), and mouse anti-Ki67 monoclonal antibody (550609, BD Sciences). The secondary antibodies, Alexa Fluor 488- or 568-conjugated anti-mouse or anti-rabbit IgG (Invitrogen), were used at a dilution of 1:500. Images were obtained using an Olympus FV1000 confocal microscope.

\section{BrdU labelling of LECs in humans}

We used BrdU labelling to identify and quantify proliferating LECs from human cadaver eyes. Whole-mount human lens capsules were pulsed with BrdU and then stained with an antibody against BrdU to determine the distribution and density of proliferating LECs. In brief, within 12-24 $\mathrm{h}$ after death, lenses from post-mortem donor eyes were obtained from the Eye Bank of Zhongshan Ophthalmic Center in Guangzhou, China. Twelve lenses in total from six donors were used for the experiment. A small puncture injury was made on the anterior surface of a post-mortem human lens using a 30-gauge needle. The lenses were cultured at $37{ }^{\circ} \mathrm{C}$ in Dulbecco modified Eagle medium (DMEM) supplemented with $10 \%$ FBS in a humidified incubator with $5 \% \mathrm{CO}$. The contralateral lens from the same donor was treated under the same conditions but did not receive a puncture injury and was used as a control. To label the proliferating LECs, both groups of lenses were incubated in $100 \mu \mathrm{g} \mathrm{ml}$

${ }^{-1} \mathrm{BrdU}$ (Sigma-Aldrich) $24 \mathrm{~h}$ after the puncture injury. The lens was then removed from the capsular bag, and the lens capsules were fixed in $4 \%$ formaldehyde and subjected to BrdU staining using a standard immunohistochemistry protocol according to the manufacturer's instructions (CST, Boston, Massachusetts). Images were taken using a Carl Zeiss microscope (Jena, Germany).

\section{Study design, execution, and oversight of clinical trial in humans}

This study was approved by the institutional review board of the Zhongshan Ophthalmic Center (ZOC). Informed written consent was obtained from the parents or guardians of the infants before enrolment, and the tenets of the Declaration of Helsinki were followed throughout the study. The study was conducted in accordance with an international guideline and protocol for visual function measurements in paediatric cataract surgery and a protocol of the Childhood Cataract Program of the Chinese Ministry of Health (CCPMOH) and had an independent data and safety monitoring board of ZOC-CCPMOH. 


\section{Description of current surgical method for cataract extraction}

The current standard-of-care treatment for paediatric cataract involves removal of the cataractous lens through a relatively large opening using anterior continuous curvilinear capsulorhexis (ACCC, about $6 \mathrm{~mm}$ in diameter, Extended Data Fig. 1), followed by cataract extraction and artificial lens implantation or placement of postoperative aphakic eyeglasses/ contact lens in paediatric cataract patients younger than two years. Some patients underwent additional posterior continuous curvilinear capsulorhexis (PCCC) and anterior vitrectomy.

\section{Establishment of a minimally invasive capsulorhexis surgery method to preserve LECs}

We established a new capsulorhexis surgery method to facilitate lens regeneration (Fig. 3a). First, we decreased the size of the capsulorhexis opening to $1.0-1.5 \mathrm{~mm}$ in diameter. This results in a minimal wound of about $1.2 \mathrm{~mm}^{2}$ in area, which is only about $4.3 \%$ the size of the wound created by the current method. Second, we moved the location of the capsulorhexis to the peripheral area of the lens instead of the central area. A $0.9 \mathrm{~mm}$ phacoemulsification probe was used to remove the lens contents and/or cortical opacities. These changes provide significant advantages. First, it considerably reduces the size of the injury, which resulted in a lower incidence of inflammation and much faster healing. Second, it moves the wound scar away from the central visual axis to the periphery, leading to improved visual axis transparency. Third, it preserves a nearly intact transparent lens capsule and layer of LECs, which have regenerative potential and are critically required for the regeneration of a natural lens.

\section{Clinical trial of minimally invasive lens surgery in human infants with congenital cataract}

The clinical trial is an open label, randomized controlled trial in a study population of paediatric cataract patients (age: 0-2 years). Except the trial participants, all other parties (care providers, outcome assessors) were blinded to treatment allocation. A clinical trial consort flowchart is listed in the Extended Data Fig. 8a. Paediatric patients were enrolled accordingly inclusion and exclusion criteria below (ClinicalTrials.gov identifier:

NCT01844258). Inclusion criteria were the following: infants were $\_4$ months old, and diagnosed with bilateral uncomplicated congenital cataract with an intact non-fibrotic capsular bag. Exclusion criteria included preoperative intraocular pressure (IOP) $>21 \mathrm{~mm}$ $\mathrm{Hg}$, premature birth, family history of ocular disease, ocular trauma, or other abnormalities, such as microcornea, persistent hyperplastic primary vitreous, rubella, or Lowe syndrome. In total, twelve paediatric cataract patients ( 24 eyes) received the new minimally invasive lens surgery (Table 1). Twenty-five paediatric cataract patients (50 eyes in total) were enrolled as the control group to receive the current standard surgical treatment (Extended Data Fig. 8a). Bilateral eye surgeries of the same patient were conducted during the same operation session.

We defined the incidence of corneal oedema as a $>5 \%$ increase in central corneal thickness one week post-surgery, and the incidence of severe anterior chamber inflammation as a Flare value $>10$ evaluated by Pentacam system (OCULUS, Germany) and Laser flare meter (KOWA FM-600, Japan). Early-onset ocular hypertension was identified as IOP >21 mm Hg by Tonopen (Reichert, Seefeld, Germany) within one month after surgery. Macular oedema was identified by fundus OCT (iVue, Optovue, Germany) as an increase in central macular 
thickness $>10 \%$ one week post-surgery. When indicated, VAO, defined by visual decline and the degree to which the fundus was obscured, was treated with YAG laser capsulotomy at follow-up.

Compared to infants operated on using our new surgical technique, infants who received the traditional technique had a higher incidence of anterior chamber inflammation one week after surgery, early-onset ocular hypertension, and increased VAO (Table 1). However, in the group treated with our new method, a transparent regenerated biconvex lens was found in $100 \%$ of eyes three months after surgery, while no regenerated biconvex lenses formed in the group treated with the standard technique. In addition, $100 \%$ of the capsular openings healed within one month after surgery in the experimental group, but no capsular openings healed in the control group.

\section{Evaluation of paediatric visual acuity}

Testing equipment included a set of Teller Acuity Cards (Vistech Consultants, Dayton, Ohio). The set of cards consists of 15 cards with gratings ranging in spatial frequency from 0.32 to 38 cycles per $\mathrm{cm}$, in half-octave steps, and one blank grey card. A 4-mm peephole in each card allows the tester to view the child's face through the card during testing. Test distance was kept constant by use of an aid to measure the distance from the child's eyes to the card throughout testing. For $38 \mathrm{~cm}$, the aid was the distance measured from the tester's elbow to a specific knuckle on the tester's hand, and for $55 \mathrm{~cm}$, the aid was the length (55 $\mathrm{cm}$ ) of the Teller Acuity Card. Testers were instructed to hold the cards without wrapping their fingers around the front side of the card, as this may attract the child's attention. Testers presented the cards directly in front of the child and observed the child either over the top of the card or through the peephole in the card.

During each acuity test, a masked visual acuity examiner was aware that the gratings were arranged in order from lower to higher spatial frequencies in halfoctave steps, but were masked to the absolute spatial frequency of the grating on each card. The subset of spatial frequencies used for each test was selected according to a pseudorandom order from among three possible subsets of spatial frequencies for the subject's age group. All three subsets for each age group included spatial frequencies known to be well above the threshold for that age group. To keep the visual acuity examiner masked to the absolute spatial frequency, the visual acuity examiner was not permitted to look at the front of the card to confirm the location of the grating. Instead, the visual acuity examiner asked an assistant to confirm the location of the grating on the card, after the visual acuity examiner had shown a card to the subject enough times to assess whether or not the subject could detect the grating. A clinical examiner was masked to the acuity results and the assigned patient group. Acuity was scored as the spatial frequency of the finest grating and was converted to log values before data analysis.

\section{Measurement of lens refractive power}

We used a handheld auto-refractometer (PlusoptiX A09, OptiMed, Sydney, Australia) to evaluate the function of the regenerated lenses according to the manufacturer's methods. 


\section{Statistical analysis}

Descriptive statistics was provided for the primary and secondary endpoints measured by intervention groups at each time point. Mean and standard deviation was reported for continuous variables and count and percentage is reported for categorical variables. To assess whether the primary outcome, decimal acuity, was significantly improved within each group, we performed the pre-post comparison between decimal acuity measured at baseline and study endpoint using paired t-tests. Normality of the data was checked and nonparametric alternatives, Wilcoxon signed-rank test is considered if the assumption was severely violated. To evaluate whether the mean response profiles in two groups were similar, we used the linear mixed-effect model taking account for the within-subjects correlation. The baseline decimal acuity was not adjusted by the model due to the homogeneity of this measurement as shown in the summary statistics. As the standard-ofcare approach requires laser surgery at 3 months while the novel treatment does not, we fit two models using before and after laser surgery data, separately, to demonstrate the superiority of the novel approach. In each model, the outcome is the decimal acuity measured at four time points: baseline, 1 week, 3 months (before or after laser surgery) and 6 months; time (baseline as the reference level), treatment assignment and their interaction are the fixed effects; and patient is the random effect. Significant associations are identified using likelihood ratio test (LRT) by comparing models with and without a fixed effect. A linear mixed-effect model is fit again by dropping out the insignificant fixed effect until the final model is selected. A contrast test is performed when necessary. For the secondary aim, we compared the proportions of each condition of complications between two groups. We assumed the occurrence of complications for eyes from the same patient were independent. The mean difference and its $95 \%$ confidence interval was reported. A two-proportion z-test was used with the nonparametric $X^{2}$ test as alternative if the normality assumption was violated. All tests were two-sided and a $P$ value less than 0.05 is considered to be statistically significant.

\section{Evaluation of accommodative response}

Accommodative response was measured by an open-field autorefractor (SRW-5001K; ShinNippon, Tokyo, Japan), which allows targets to be viewed at any distance. The paediatric patients were positioned for autorefractor measurement with assistance from their parents. The patients were guided to fixate binocularly at a near target $(33 \mathrm{~cm}, 5 \times 5$ array of smiley faces of N10 size) and a far target ( $3 \mathrm{~m}, 5 \times 5$ array of smiley faces of N10 size) by a trained and certified investigator or study coordinator. The measurements from non-cycloplegic autorefraction were performed three times at each target distance by the same trained and certified investigator throughout the study, in order to maintain accuracy and consistency throughout the trial. Measurements were taken in the same quiet environment with consistent room illumination to diminish the influence of distracting factors and to maintain subjects' concentration. The spherical equivalent refractive value (SER) was recorded for each measurement and the mean value was calculated for evaluation of an accommodative response. The value of accommodative response was the difference between SER values for the near and the far target. We also used dynamic retinoscopy to measure the infants' accommodation ${ }^{29-31}$. In brief, we recorded a lens dioptre value using retinoscopy when a patient was guided to fixate on a target $3 \mathrm{~m}$ away. Then another lens dioptre value was 
recorded when the target was moved closer, at a distance of $33 \mathrm{~cm}$ from the eyes. The difference between these two measurements was used to evaluate lens accommodative power.

\section{Extended Data}

A

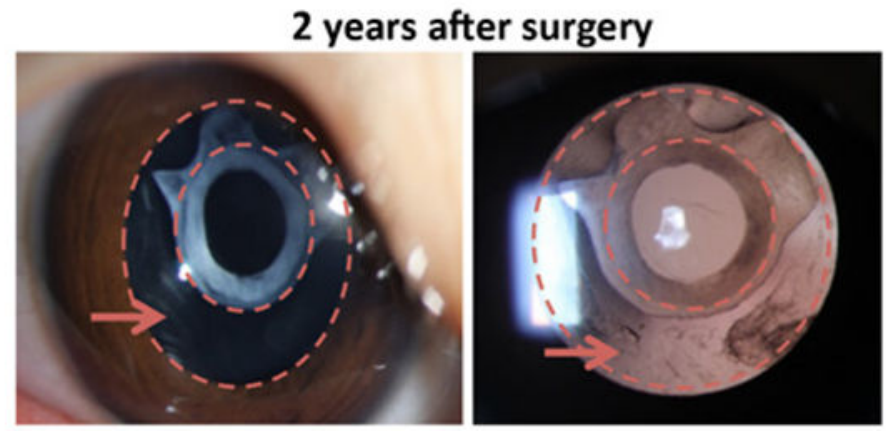

B

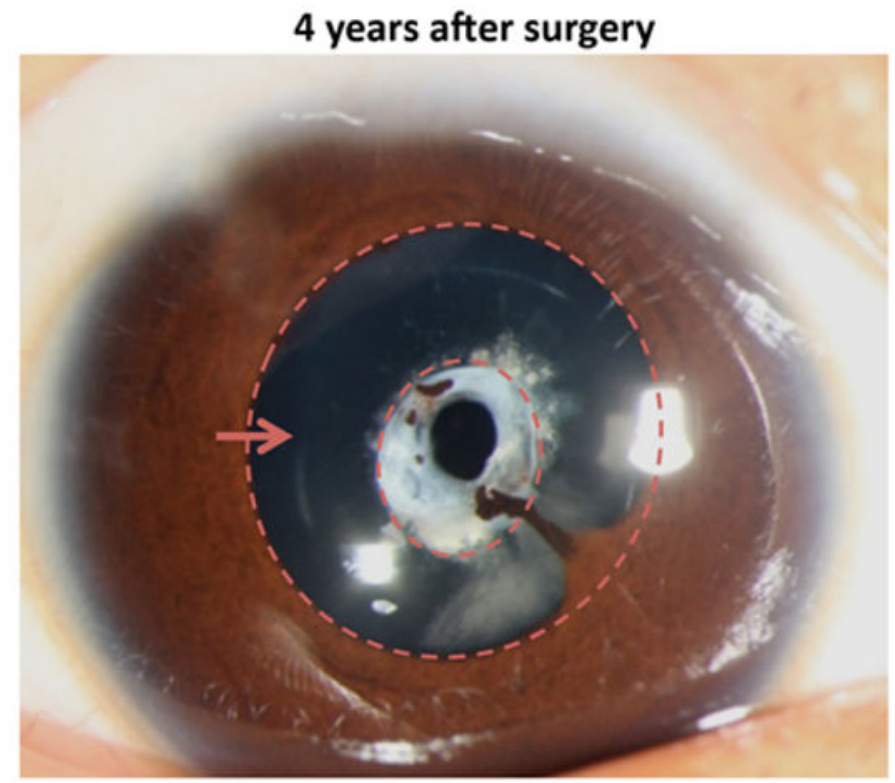

C

\section{Current Pediatric Cataract Surgery}

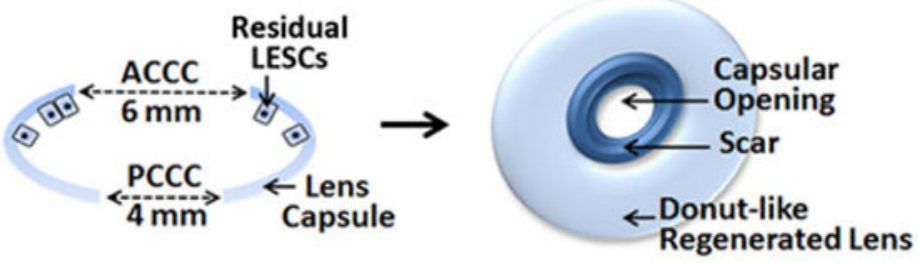

Extended Data Figure 1. Surgical methods and lens regeneration for congenital cataract a, b, Slit-lamp photography of 'doughnut-like' lens regeneration at different time points after treatment using the current surgical method. Two years after surgery (a), the 
transparent regenerated lens tissue contained the sealed capsular opening with an opaque white scar at the centre. The regions between the dashed circles indicated by the red arrows are the regenerated lens tissues. Four years after surgery (b), the capsular opening was constricted compared to that seen at two years post-surgery, indicating continued growth of the regenerated lens. There was also the complication of iridolenticular synechiae. c, Schematic diagrams of the current surgical method for paediatric cataracts: the currently practiced paediatric ACCC creates an opening $6 \mathrm{~mm}$ in diameter at the centre of the anterior capsule, removing the LECs underneath it and leaving a relatively large wound area of 28 $\mathrm{mm}^{2}$. The scars formed often cause postoperative VAO. Additionally, PCCC and anterior vitrectomy are commonly performed at follow-up visits.
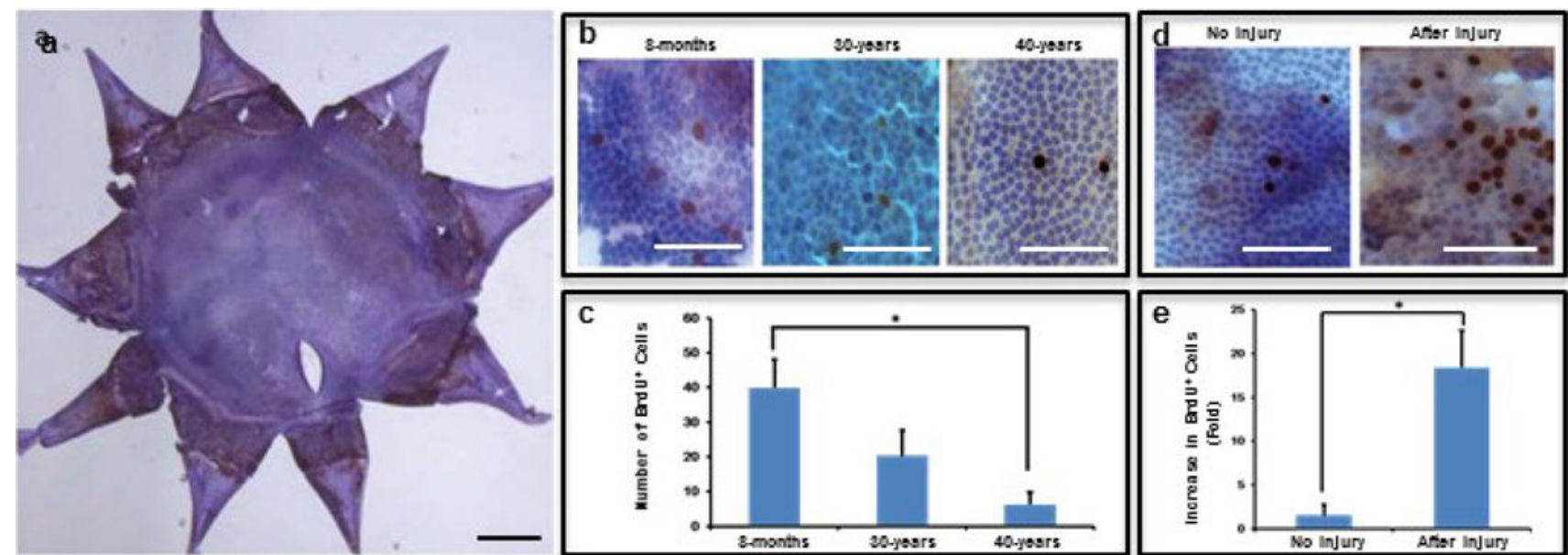

f
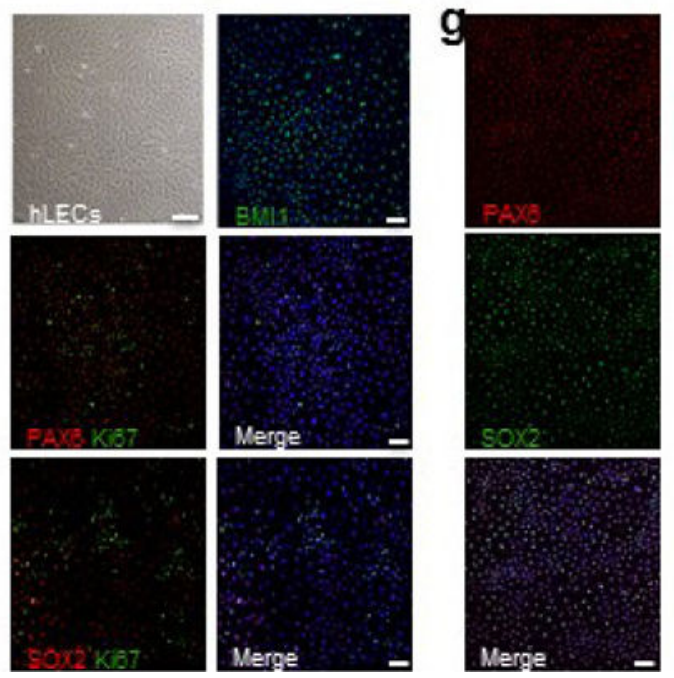

Extended Data Figure 2. BrdU pulse labelling of human LECs

a, Whole mount of a human lens capsule showing BrdU ${ }^{+}$cells (brown) by enzymatic immunohistology and diaminobenzidine staining. b, High-magnification images of human donor lenses showing BrdU ${ }^{+}$LECs. c, Bar graph showing quantification of $\mathrm{BrdU}^{+}$cells. There was an age-dependent decrease in the number of BrdU ${ }^{+}$cells ( 8 months, $39.9 \pm 8.1$; 30 years, $20.3 \pm 7.3$ and 40 years, $5.9 \pm 2.9 ; 8$ months versus 40 years, $* P<0.05)$. Six 
randomly chosen fields of each capsule were used for analysis, four samples in each group, ( $n=24$ fields, chosen over four samples). d, High-magnification images of whole-mount staining of human lens capsules with or without injury showed a marked increase in the number of $\mathrm{BrdU}^{+}$cells after injury. $\mathbf{e}, \mathrm{Bar}$ graph showing quantification of $\mathrm{BrdU}^{+}$cells. The contralateral eyes from the respective donors were used as controls. There was a significant increase in number of BrdU ${ }^{+}$cells. No injury, $1.5 \pm 1.2$; after injury, $18.4 \pm 4.2$; fold change after injury, $11.3 \pm 2.5 ; * P<0.05$. Six randomly chosen fields within the germinative zone of each capsule were used for analysis, five samples in each group $(n=30$ fields, chosen over five samples). Data shown as means \pm s.d. f, Cultured human fetal LECs were positive for BMI-1 (green, right upper panel); co-staining of PAX6 (red) and Ki67 (green), middle panels; co-staining of SOX2 (red) and Ki67 (green), lower panels. g, Co-staining of PAX6 (red) and SOX2 (green) of human fetal LECs. All scale bars, $100 \mu \mathrm{m}$.

a

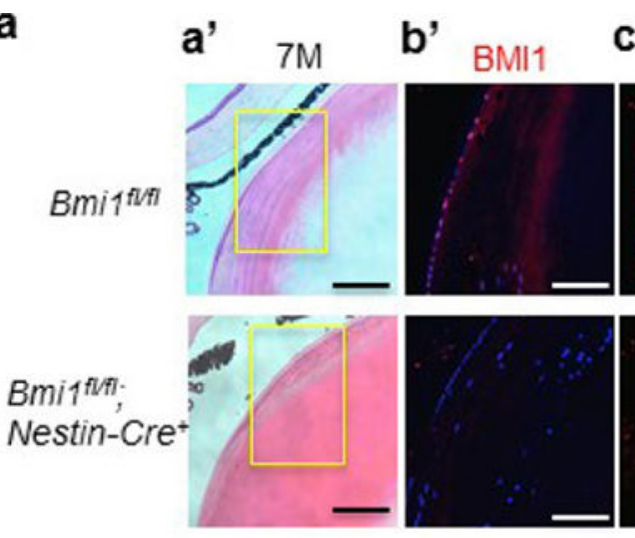

b

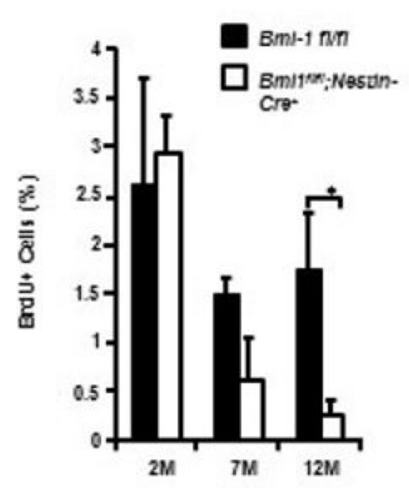

C
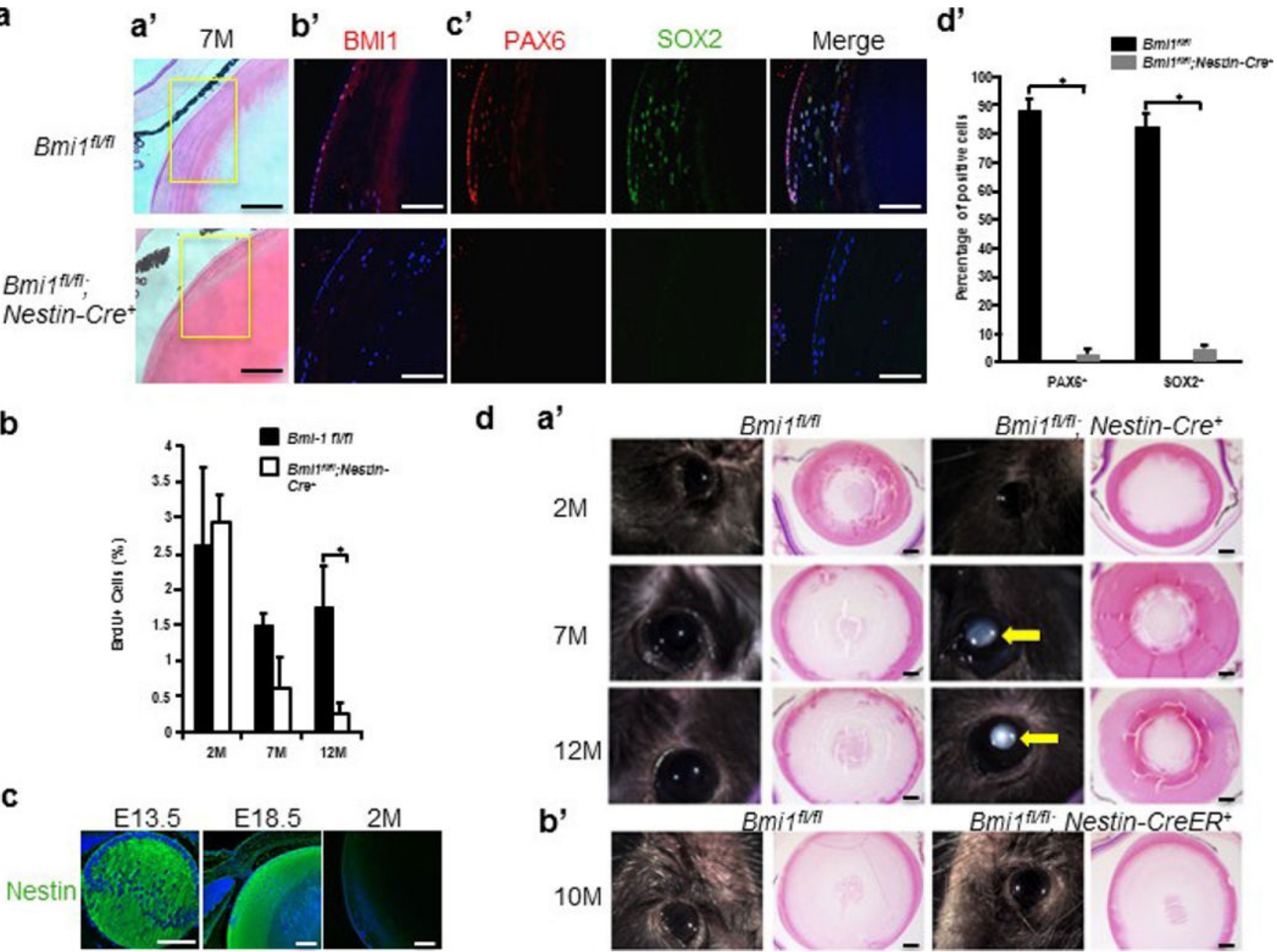

Bmitilit

$2 \mathrm{M}$

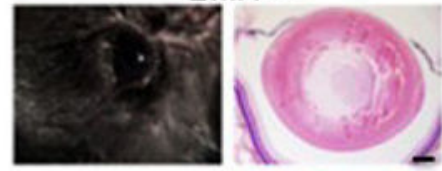

Bmi $1^{\text {fint }}$ : Nestin-Cre+

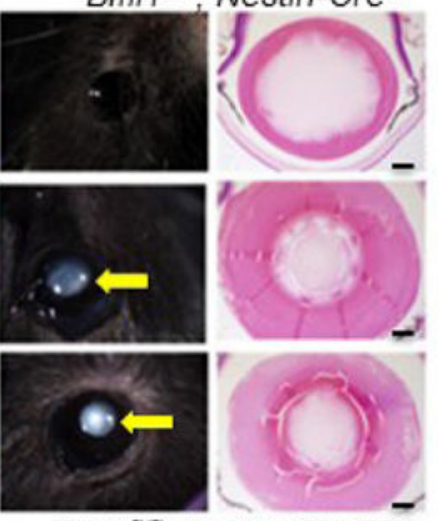

Bmi1 fillit: Nestin-CreER+

b'

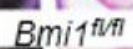

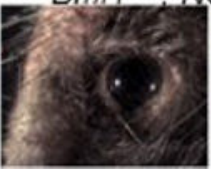

Extended Data Figure 3. Conditional deletion of Bmi-1 led to decrease in $\mathrm{Pax6}^{+}$and Sox2 ${ }^{+}$cells and cataract formation

A, Loss of Bmi- 1 reduced the $\mathrm{Pax}^{+}$and Sox $2^{+}$LECs population. a, Representative images of haematoxylin and eosin-stained lens sections from Bmifl/ffl control mice and Nestincre;Bmi $1^{f l / f l}$ mice. b, Representative images of Bmi-1 (red) staining in LECs. c, Pax6 (red) 
and Sox2 (green) immunostaining. d, Percentage of Pax6 $6^{+}\left(\right.$Bmil $^{f l / f 1}, 88.5 \pm 2.9 \%$; Nestincre;Bmi $\left.{ }^{f l / f l}, 2.4 \pm 2.3 \%\right)$ and Sox $2^{+}\left(\right.$Bmil $^{f l / f l}, 82.7 \pm 3.9 \% ;$ Nestin-cre;Bmil ${ }^{f l / f l}, 4.9$ $\pm 1.5 \%$ ) cells ( $n=5$ mice; 5 sections counted per mice, for a total of 25 sections across 5 mice), $* P<0.001$. Data are shown as mean \pm s.d. B, Conditional deletion of Bmil led to reduced LEC proliferation. The percentage of BrdU ${ }^{+}$LECs per eye is shown $\left(2 \mathrm{M}: B m i 1^{f l / f 1}\right.$, $2.6 \pm 0.9 \%$; Nestin-cre;Bmil ${ }^{f l / f 1}, 3.0 \pm 0.4 \% ; n=4$ mice. $7 \mathrm{M}:$ Bmil $^{\mathrm{fl} / f 1}, 1.5 \pm 0.2 \%$; Nestincre;Bmi1 ${ }^{f l / f l}, 0.6 \pm 0.4 \% ; n=6$ mice. $12 \mathrm{M}:$ Bmi1 $^{\mathrm{fl} / \mathrm{fl}}, 1.8 \pm 0.6 \%$; Nestin-cre;Bmi1 $1^{\mathrm{fl} / f 1}, 0.2$ $\pm 0.2 \% ; n=8$ mice), two sections counted per eye. Statistical significance was assessed using a two-tailed Student's t-test. ${ }^{*} P<0.05$. Data are shown as mean \pm s.d. C, Nestin (green) staining is shown in E13.5, E18.5, and 2-month-old wild-type mice. All scale bars, $100 \mu \mathrm{m}$. D, Representative images of lenses from Nestin-cre;Bmi $1^{f l / f 1}$ and $B m i 1^{f l / f 1}$ control mice. a, Cataracts are evident in 7- and 12-month-old Nestin-cre;Bmi ${ }^{\text {fl/fl }}$ mice (arrow). b, Deletion of Bmi-1 at 6 weeks of age with Nestn-creER did not recapitulate the cataract phenotype 10 months after tamoxifen treatment. Haematoxylin and eosin-stained sections of the same eyes are also shown. All scale bars, $100 \mu \mathrm{m}$.

a
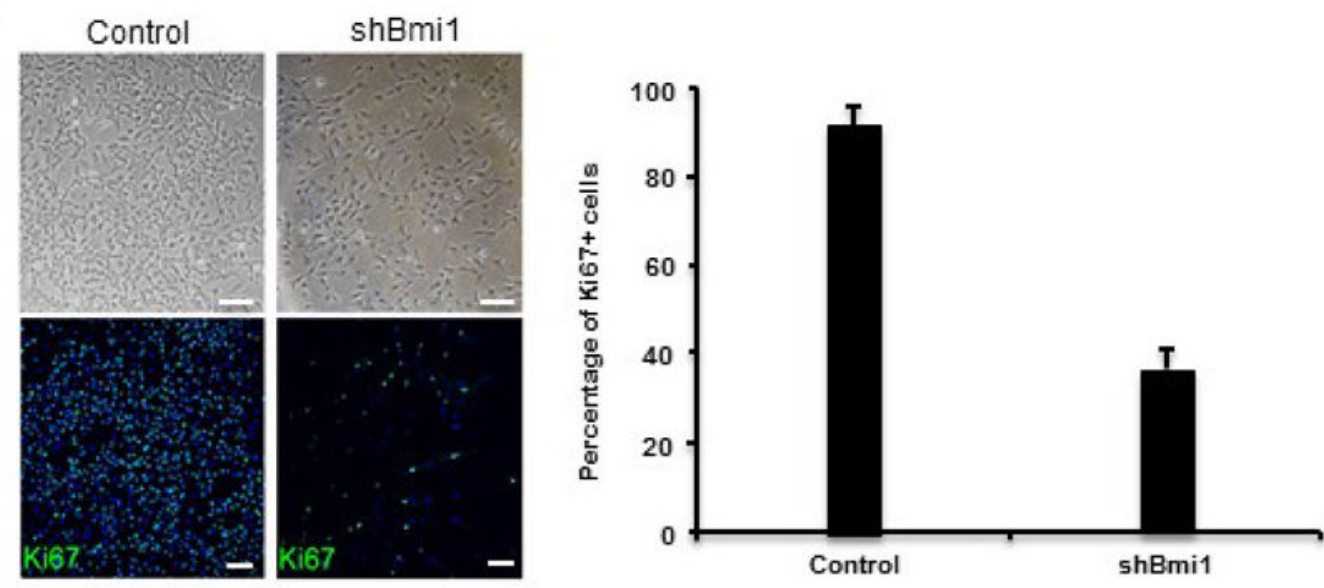

b

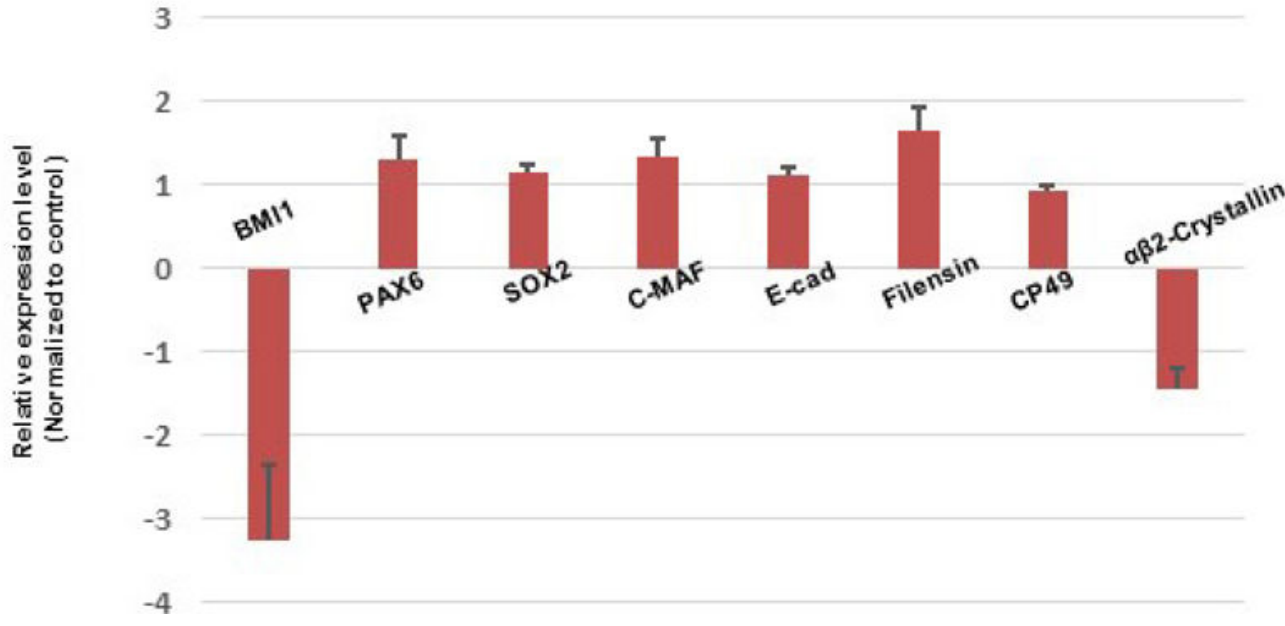

Extended Data Figure 4. Loss of BMI-1 decreased the proliferative ability of LECs

Nature. Author manuscript; available in PMC 2018 July 26. 
a, Phase-contrast photographs of human LECs (upper panels) and quantification of Ki67 proliferating human fetal LECs upon BMI1 knockdown (shBMI1) compared to controls (two shRNAs gave similar results; $n=5, P<0.05$ ). Data shown as mean \pm s.d. Blue indicates DAPI staining. $\mathbf{b}$, Loss of BMI-1 did not significantly affect expression of LEC or lens fibre cell makers in LECs. BMI1 was reduced by 3.3-fold (all $n=3, P<0.05$ ); gene expression changes of LEC markers were: 1.3-fold increase (PAXG), 1.1-fold increase (SOX2), 1.3-fold increase (C-MAF) and 1.1-fold increase (E-cadherin); gene expression changes of differentiated lens fibre cell markers were: 1.6-fold increase (Filensin), 0.9 fold increase (CP49) and 1.4-fold decrease (CRYBA2). Two different shRNAs gave similar results; $n=5, P<0.05$. Data shown as mean \pm s.d.
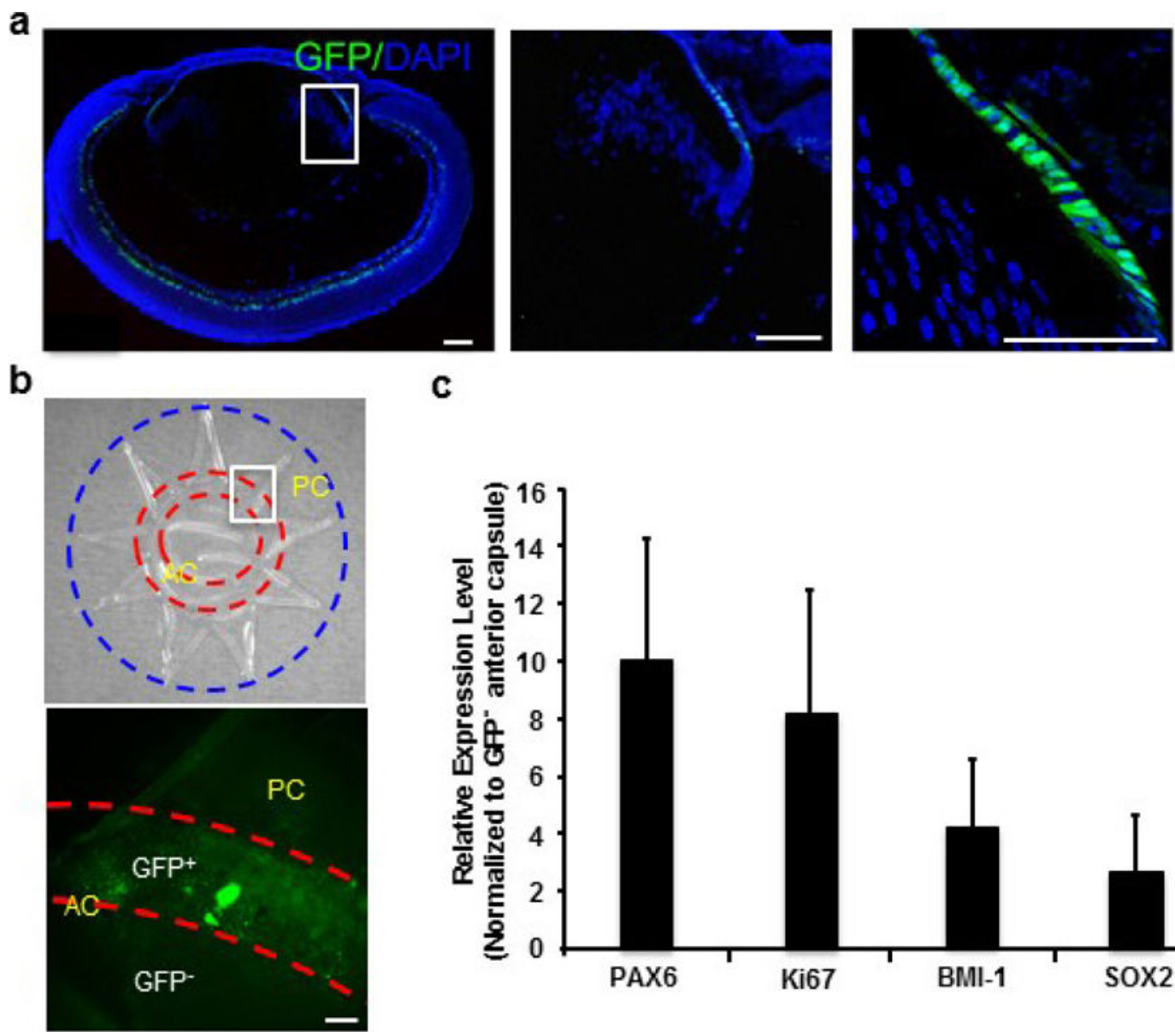

C

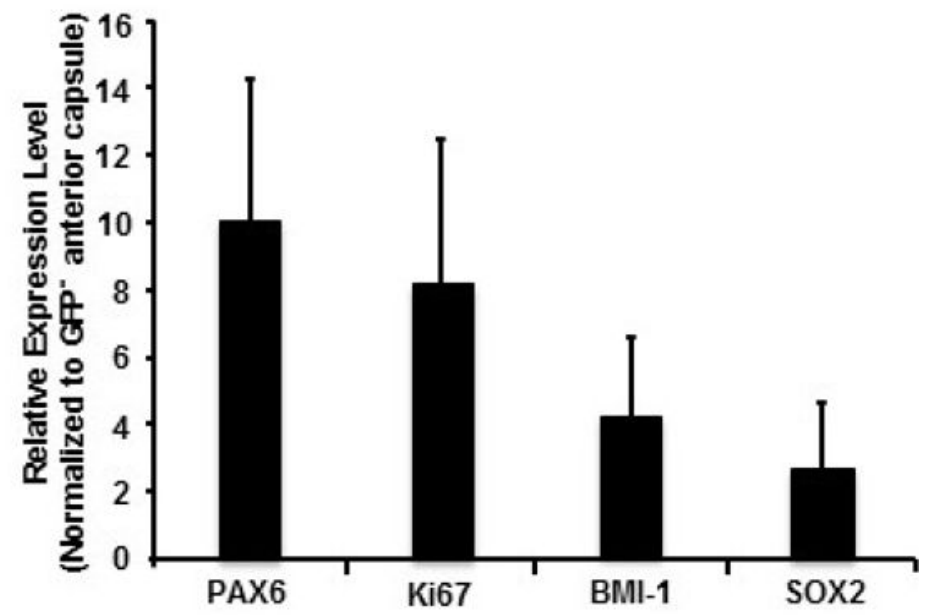

Extended Data Figure 5. Higher expression levels of Bmil, Sox2 and Ki67 in Pax6 ${ }^{+}$LECs a, Pax6-GFP ${ }^{+}$LECs were observed at the germinative zone. Left panel, a section of lens of a Pax6P0-3.9-GFPcre mouse at P1. Middle and right panels, higher magnification of the framed area in the left panel. Blue indicates DAPI staining. b, Upper panel, bright-field photograph showing flat-mount preparation of a lens capsule of a Pax6P0-3.9-GFPcre mouse at 6 months; lens capsule materials between two red circles were dissected to enrich Pax6-GFP ${ }^{+}$LECs. Lower panel, fluorescence image of GFP $^{+}$LECs from the framed area in the upper panel. AC, anterior capsule; PC, posterior capsule. c, Comparison of gene expression levels in Pax6-GFP ${ }^{+}$LECs versus GFP $^{-}$LECs in anterior lens capsule in 6- 
month-old mice, increased expression of the following genes were observed: 10.1-fold in Pax6 (P < 0.005), 8.2-fold in Ki67 ( $<<0.05)$, 4.3-fold in Bmi1 $(P<0.05)$, and 2.6-fold in Sox2 $(P<0.05)$, all $n=5$. Data shown as mean \pm s.d.
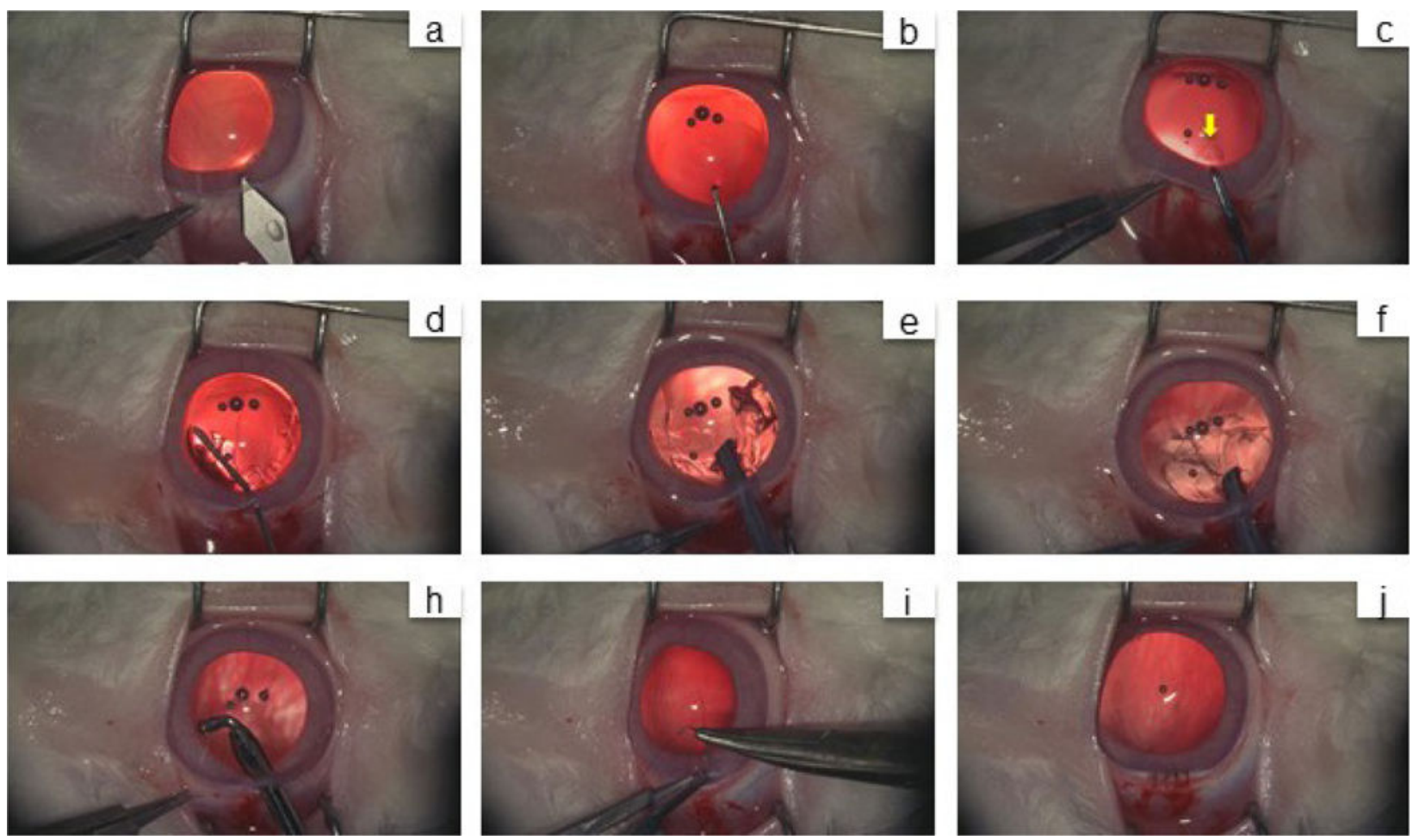

Extended Data Figure 6. Lens regeneration surgery in rabbits

a, A 3.2-mm keratome was used to make a limbus tunnel incision at the 11-12 o'clock position into the anterior chamber. $\mathbf{b}$, The capsular opening was created by a capsulorhexis needle. c, A 1-2 mm diameter anterior capsulotomy was performed using the anterior continuous curvilinear capsulorhexis (ACCC) technique near the capsular opening area (yellow arrow). d, A blunt needle was used to inject balanced salt solution for hydrodissection of the cortex from the anterior capsule. e, The cortex was removed using a phacoemulsification device. $\mathbf{f}$, The remaining cortex was removed using irrigation and aspiration. $\mathbf{h}$, An elbow I/A handle was used to clear the equatorial cortex. $\mathbf{i}, \mathbf{j}$, The limbus wound was sutured with an interrupted 10-0 nylon suture. The wound was found to be watertight. 


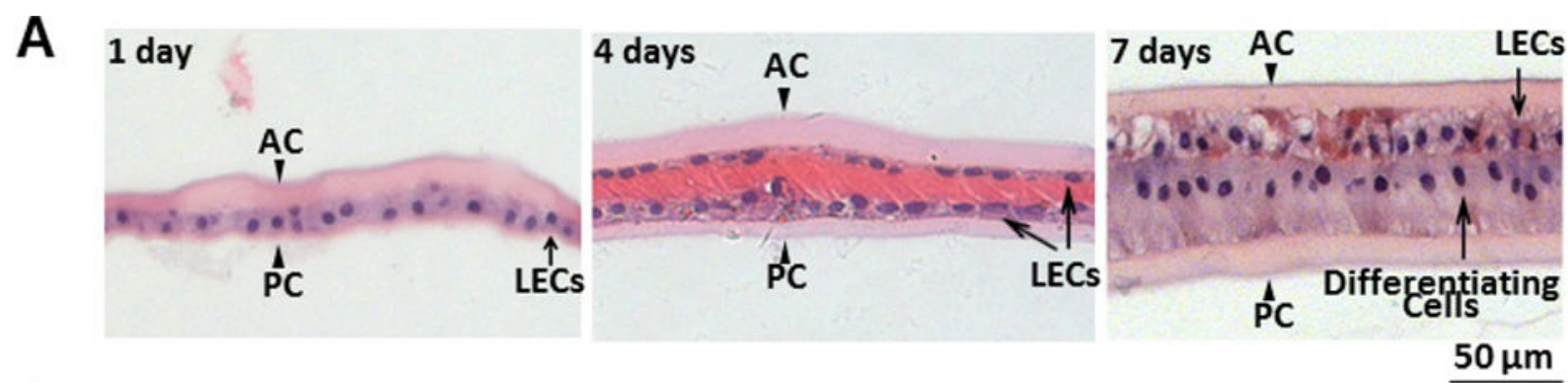

B

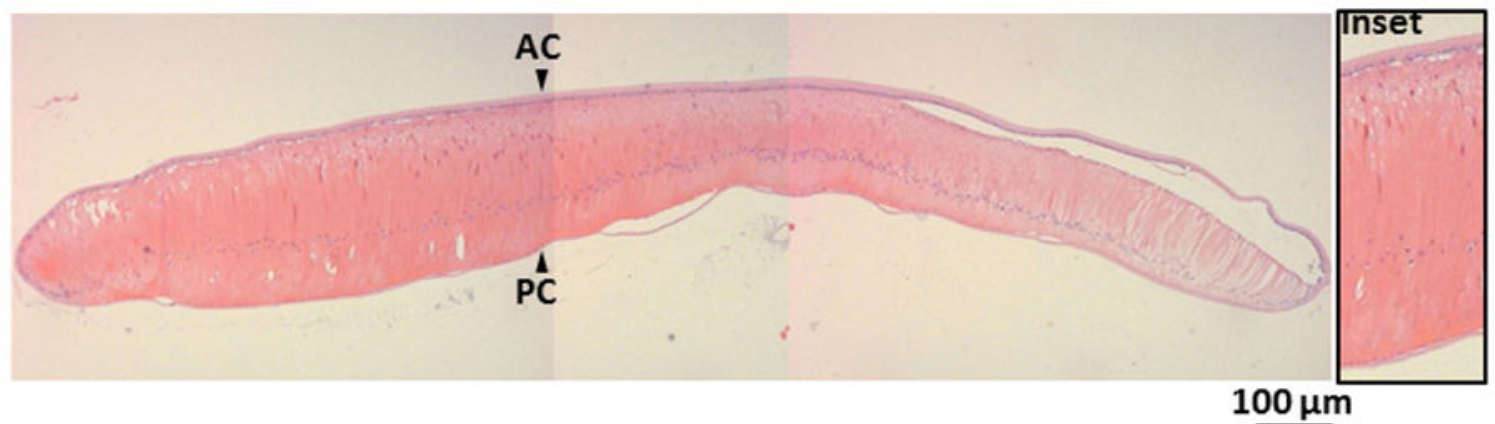

C

Day 1
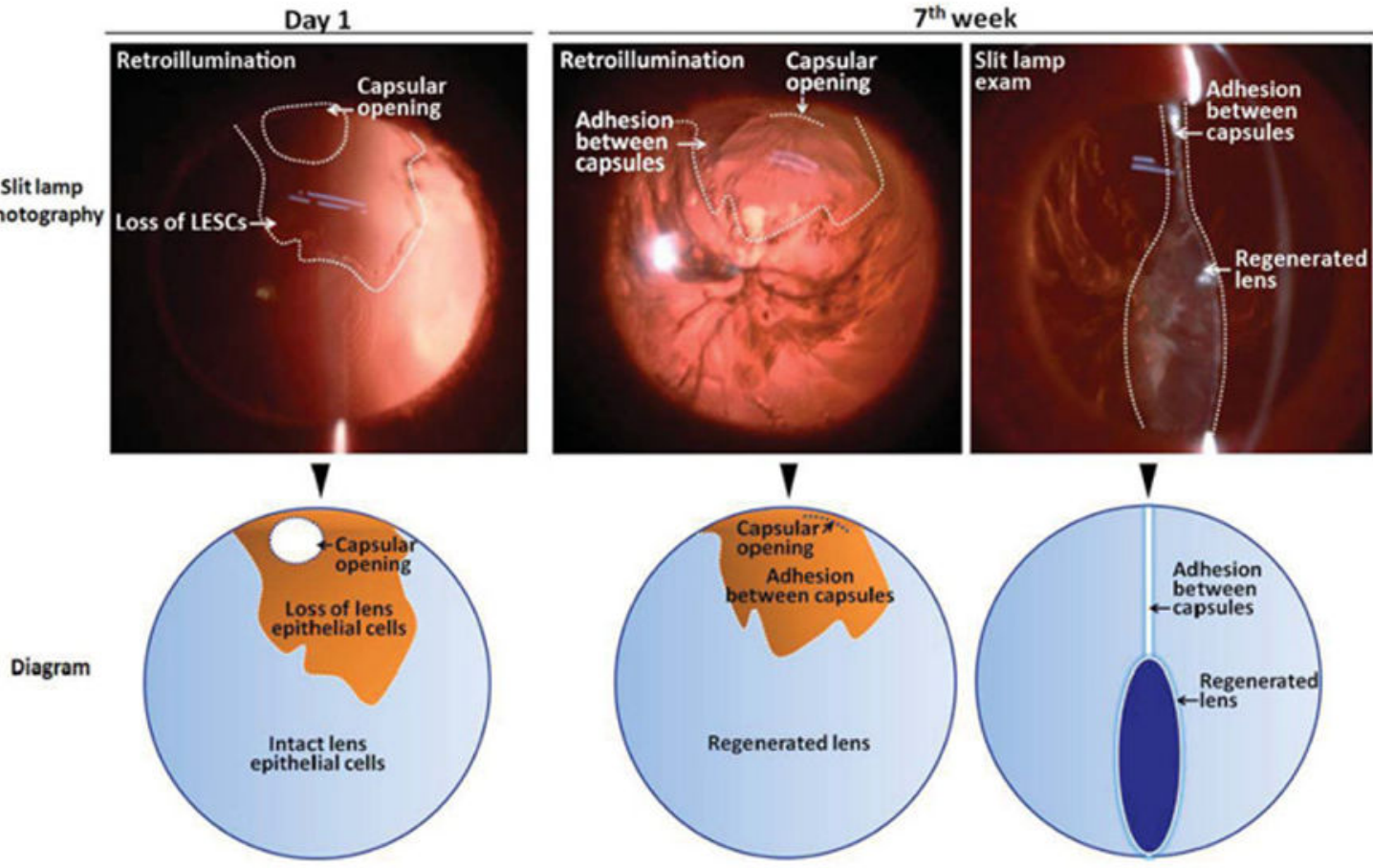

Extended Data Figure 7. Lens regeneration in rabbits

a, Haematoxylin and eosin staining of regenerated lenses at different time points after surgery. At postoperative day 1, a monolayer of LECs between the anterior and posterior capsules was visible (arrowheads). At postoperative day 4, LECs proliferated and covered the posterior capsule. At postoperative day 7, LECs in the posterior capsule began to elongate and differentiate. b, At postoperative day 28, LECs in the posterior capsule further elongated, forming primary lens fibres. c, Transparency and shape of regenerated lenses in rabbits. Upper panel, slit-lamp photography of a regenerated lens at different time points 
after surgery. Lower panel, schematic diagram of slit-lamp photographs in the upper panel. At day 1 after surgery, the capsular opening was clearly seen in the peripheral anterior capsule, and the area of LEC loss during surgery is indicated. At 7 weeks after surgery, loss of LECs led to adhesion between the anterior and the posterior capsule and inhibition of lens regeneration in this area.
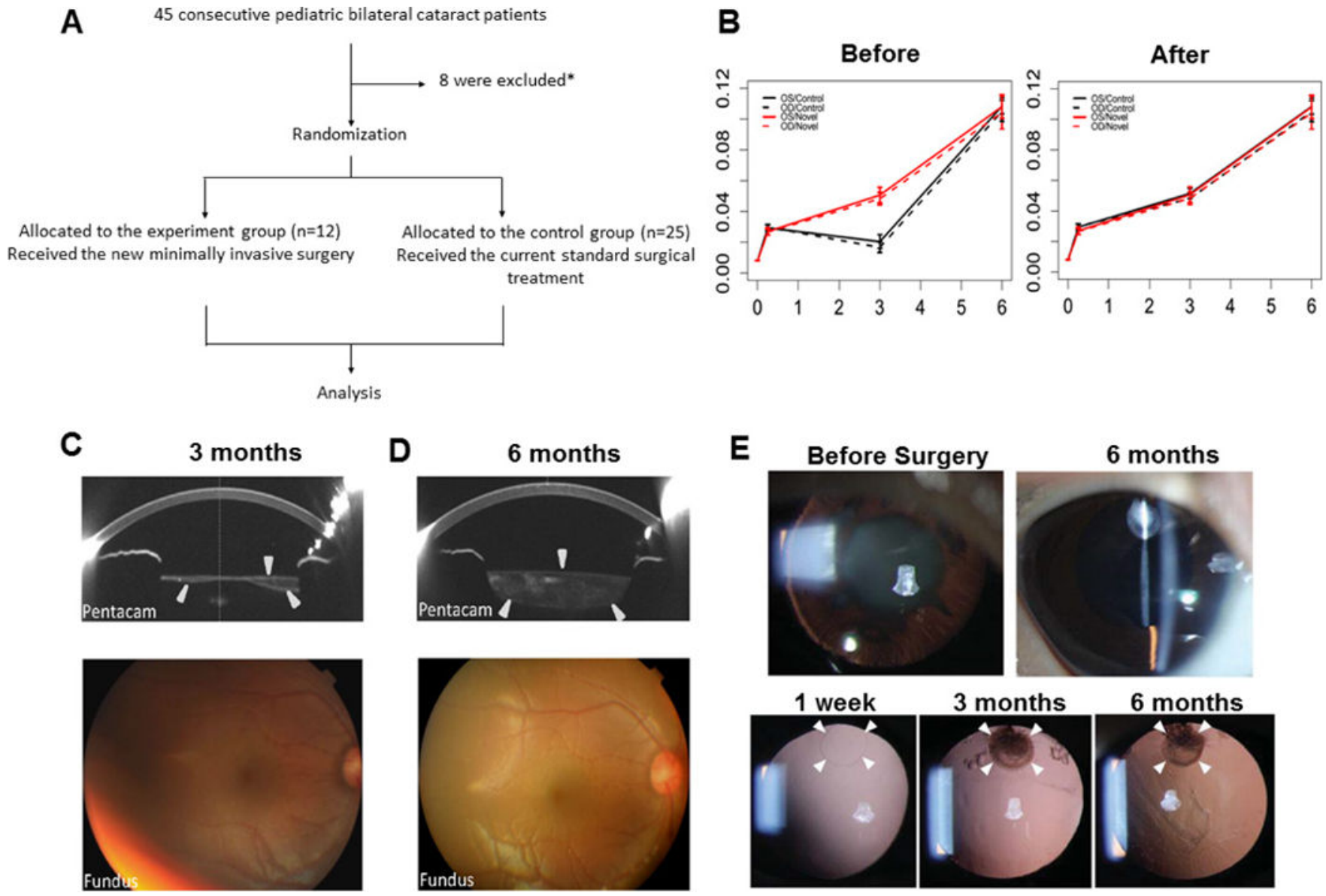

\section{E}
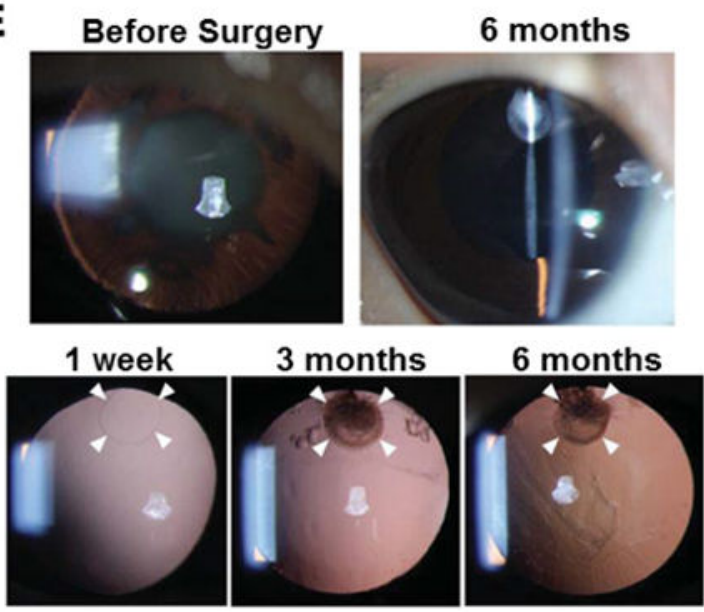

Extended Data Figure 8. Human lens regeneration

a, A clinical trial consort flowchart. $\mathbf{b}$, Comparison of visual acuity mean response profiles in two groups. A non-parallel pattern of mean responses between two groups was observed largely due to the vision loss at 3 months before laser surgery in the control group (left panel), whereas a parallel pattern of mean responses between two groups was observed using time points including 3 months after laser surgery (right panel); $n=25$ control, $n=12$ experimental. Data are shown as mean \pm s.d. c, Lens thickness increased after surgery. Pentacam showed that 3 months after surgery, the regenerating lens tissue grew from the periphery of the capsular bag to the centre. The sealed capsular bag was only partially filled, appearing spindle-shaped on cross-sectional scan. The fundus was clearly visible on ophthalmoscopy. Arrowheads indicate the regenerated lens structure. d, Six months after surgery, the capsular bag was filled with regenerated lens tissue and appeared biconvex on cross-sectional scan by Pentacam. The anterior-posterior capsular adhesion disappeared. The fundus could be seen clearly using an ophthalmoscope with an 18-dioptre lens. e, Minimally 
invasive capsulorhexis preserved LECs for lens regeneration in human infants. Top panel, slit-lamp exam demonstrating human infant's eye visual axis transparency 6 months after minimally invasive surgery compared to baseline (before cataract surgery). Bottom panel, retro-illumination demonstrating the reduced size of the capsulorhexis (white arrowheads).

\section{Extended Data Table 1 Clinical Outcome Analysis}

Extended Data Table 1a. Linear mixed-effect model with decimal acuity as outcome; time, treatment and their interaction as fixed effects; and patient as random effect.

Extended Data Table 1b. Linear mixed-effect model with decimal acuity as outcome; time and treatment as fixed effect; and patient as random effect.

Extended Data Table 1c. Likelihood ratio test of fixed effects based on the analysis of response profiles.

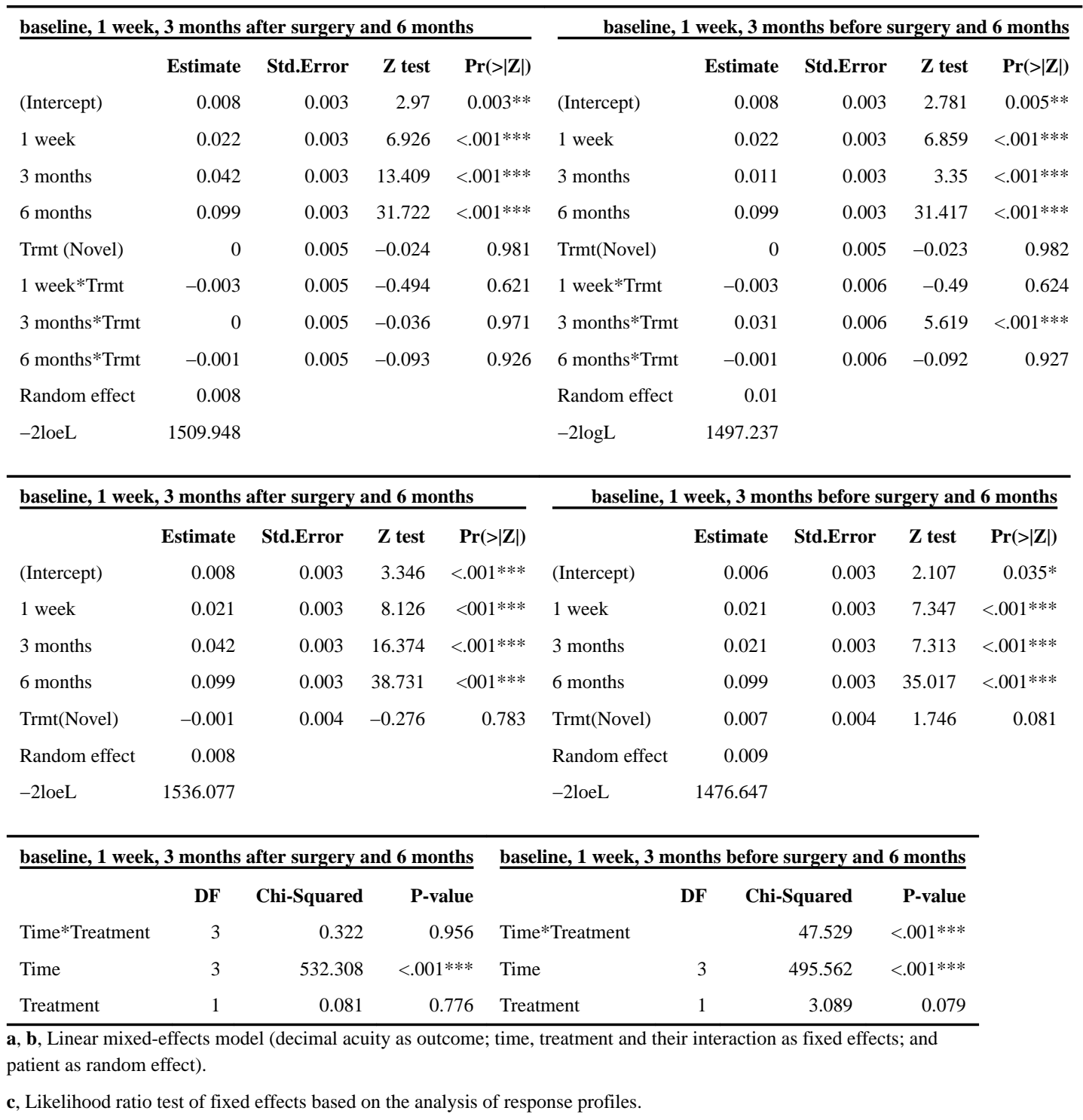

Nature. Author manuscript; available in PMC 2018 July 26. 


\section{Supplementary Material}

Refer to Web version on PubMed Central for supplementary material.

\section{Acknowledgments}

We thank E. Yeh, L. Xi, J. Shelton, A. Pineda and R. Ufret-Vincenty for technical assistance. This study was funded by 973 Program (2015CB964600, 2014CB964900, 2013CB967504); a Major International Joint Research Project (No. 81320108008); 863 Program (2014AA021604), NSFC (No. 81270981); the State Key Laboratory of Ophthalmology, Zhongshan Ophthalmic Center, Sun Yat-sen University; Research to Prevent Blindness and the Howard Hughes Medical Institute.

\section{References}

1. Barbosa-Sabanero K, et al. Lens and retina regeneration: new perspectives from model organisms. Biochem J. 2012; 447:321-334. [PubMed: 23035979]

2. Tsonis PA, Del Rio-Tsonis K. Lens and retina regeneration: transdifferentiation stem cells and clinical applications. Exp Eye Res. 2004; 78:161-172. [PubMed: 14729349]

3. Gwon A. Lens regeneration In mammals: a review. Surv Ophthalmol. 2006; 51:51-62. [PubMed: 16414361]

4. Gwon AE, Gruber LJ, Mundwiler KE. A histologic study of lens regeneration in aphakic rabbits. Invest Ophthalmol Vis Sci. 1990; 31:540-547. [PubMed: 2318593]

5. Stevens GA, et al. Global prevalence of vision impairment and blindness: magnitude and temporal trends, 1990-2010. Ophthalmology. 2013; 120:2377-2384. [PubMed: 23850093]

6. Lois N, Taylor J, McKinnon AD, Forrester JV. Posterior capsule opacification in mice. Arch Ophthalmol. 2005; 123:71-77. [PubMed: 15642815]

7. Visser N, Bauer NJ, Nuijts RM. Toric intraocular lenses: historical overview, patient selection, IOL calculation, surgical techniques, clinical outcomes, and complications. J Cataract Refract Surg. 2013; 39:624-637. [PubMed: 23522584]

8. Mamalis N, Davis B, Nilson CD, Hickman MS, Leboyer RM. Complications of foldable intraocular lenses requiring explantation or secondary intervention -2003 survey update. J Cataract Refract Surg. 2004; 30:2209-2218. [PubMed: 15474838]

9. Jacobi PC, Dietlein TS, Konen W. Multifocal intraocular lens implantation in pediatric cataract surgery. Ophthalmology. 2001; 108:1375-1380. [PubMed: 11470687]

10. Bothun ED, et al. One-year strabismus outcomes in the Infant Aphakia Treatment Study. Ophthalmology. 2013; 120:1227-1231. [PubMed: 23419803]

11. Infant Aphakia Treatment Study Group. A randomized clinical trial comparing contact lens with intraocular lens correction of monocular aphakia during infancy: grating acuity and adverse events at age 1 year. Arch Ophthalmol. 2010; 128:810-818. [PubMed: 20457949]

12. Beebe DC, Holekamp NM, Shui YB. Oxidative damage and the prevention of age-related cataracts. Ophthalmic Res. 2010; 44:155-165. [PubMed: 20829639]

13. Park IK, et al. Bmi-1 is required for maintenance of adult self-renewing haematopoietic stem cells. Nature. 2003; 423:302-305. [PubMed: 12714971]

14. Lessard J, Sauvageau G. Bmi-1 determines the proliferative capacity of normal and leukaemic stem cells. Nature. 2003; 423:255-260. [PubMed: 12714970]

15. Molofsky AV, et al. Bmi-1 dependence distinguishes neural stem cell self-renewal from progenitor proliferation. Nature. 2003; 425:962-967. [PubMed: 14574365]

16. TsonisPA. Animal models in eye research1st. Academic Press; 2008

17. Gogate $\mathrm{P}$, Kalua K, Courtright P. Blindness in childhood in developing countries: time for a reassessment? PLoS Med. 2009; 6:e1000177. [PubMed: 19997501]

18. WilsonME, , SaundersRA, , TrivediRH. Pediatric Ophthalmology: Current Thought and A Practical GuideSpringer-Verlag; Berlin: 2009

19. You C, et al. Visual impairment and delay in presentation for surgery in chinese pediatric patients with cataract. Ophthalmology. 2011; 118:17-23. [PubMed: 20709402] 
20. WilsonME, , TrivediRH, , PandeySK. Pediatric Cataract Surgery: Techniques, Complications, and ManagementLippincott Williams \& Wilkins; 2005

21. Zheng Q, et al. Vitreous surgery for macular hole-related retinal detachment after phacoemulsification cataract extraction: 10-year retrospective review. Eye Lond. 2012; 26:10581064. [PubMed: 22595907]

22. Zhou M, Leiberman J, Xu J, Lavker RM. A hierarchy of proliferative cells exists in mouse lens epithelium: implications for lens maintenance. Invest Ophthalmol Vis Sci. 2006; 47:2997-3003. [PubMed: 16799045]

23. Plager DA, et al. Complications, adverse events, and additional intraocular surgery 1 year after cataract surgery in the Infant Aphakia Treatment Study. Ophthalmology. 2011; 118:2330-2334. [PubMed: 21925737]

24. Nihalani BR, VanderVeen DK. Technological advances in pediatric cataract surgery. Semin Ophthalmol. 2010; 25:271-274. [PubMed: 21091011]

25. Gwon A, Gruber LJ, Mantras C. Restoring lens capsule integrity enhances lens regeneration in New Zealand albino rabbits and cats. J Cataract Refract Surg. 1993; 19:735-746. [PubMed: 8271170]

26. Rowan $\mathrm{S}$, et al. Notch signaling regulates growth and differentiation in the mammalian lens. Dev Biol. 2008; 321:111-122. [PubMed: 18588871]

27. Mich JK, et al. Prospective identification of functionally distinct stem cells and neurosphereinitiating cells in adult mouse forebrain. Elife. 2014; 3:e02669. [PubMed: 24843006]

28. Tronche F, et al. Disruption of the glucocorticoid receptor gene in the nervous system results in reduced anxiety. Nature Genet. 1999; 23:99-103. [PubMed: 10471508]

29. Gabriel GM, Mutti DO. Evaluation of infant accommodation using retinoscopy and photoretinoscopy. Optom Vis Sci. 2009; 86:208-215. [PubMed: 19165126]

30. Hainline L, Riddell P, Grose-Fifer J, Abramov I. Development of accommodation and convergence in infancy. Behav Brain Res. 1992; 49:33-50. [PubMed: 1388799]

31. Banks MS. The development of visual accommodation during early infancy. Child Dev. 1980; 51:646-666. [PubMed: 7418504] 
a
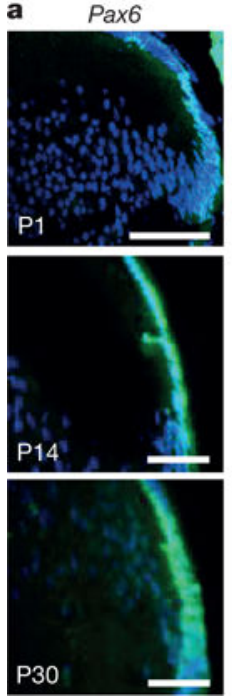

b
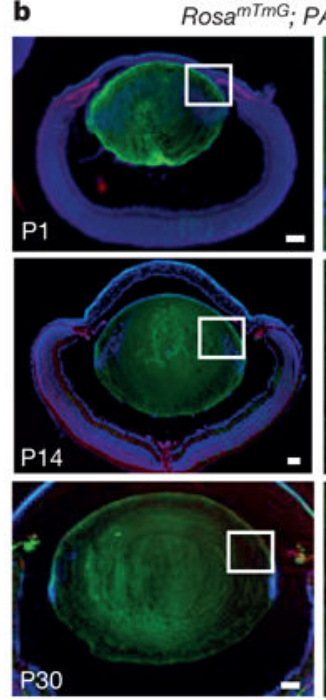
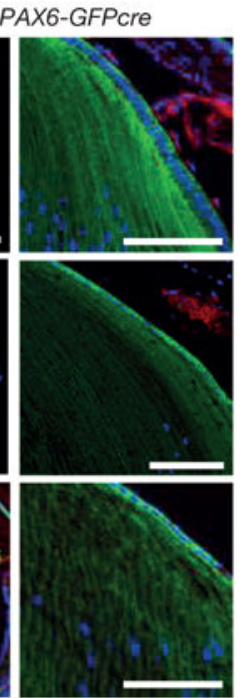

C
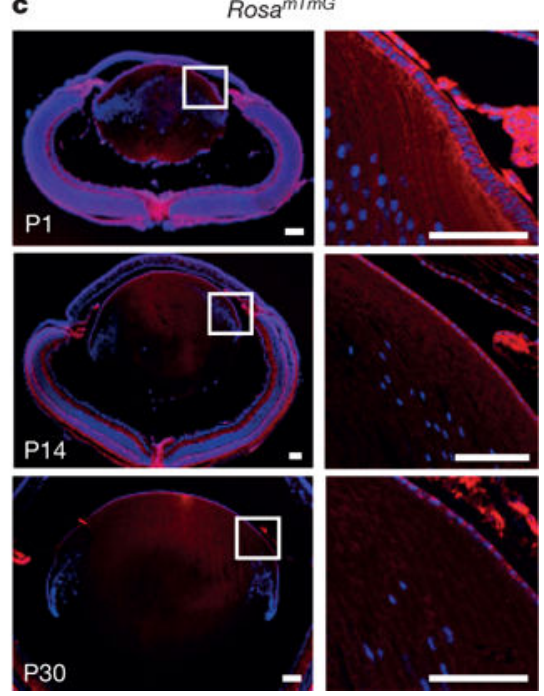

Figure 1. Lineage tracing of $\mathrm{Pax6}^{+}$LECs in mice

a, Pax6-directed GFP was expressed in mouse LEC nuclei at post-natal days P1, P14 and P30; a sagittal section of a P0-3.9-GFPcre mouse lens is shown. Blue and green represent DAPI and GFP, respectively. b, Lineage tracing of Pax6 $6^{+}$LECs in $R O S A^{m T m G}$; P0-3.9GFPcre mice at P1, $\mathrm{P} 14$ and $\mathrm{P} 30$ reveals that lens fibre cells express membrane GFP fluorescence; hence, $\mathrm{PAX6}^{+}$LECs were able to generate lens fibre cells. c, As an additional control, the $R O S A^{m T m G}$ allele alone exhibits Tomato (red) staining at sites of nonrecombination. All scale bars, $100 \mu \mathrm{m}$. 


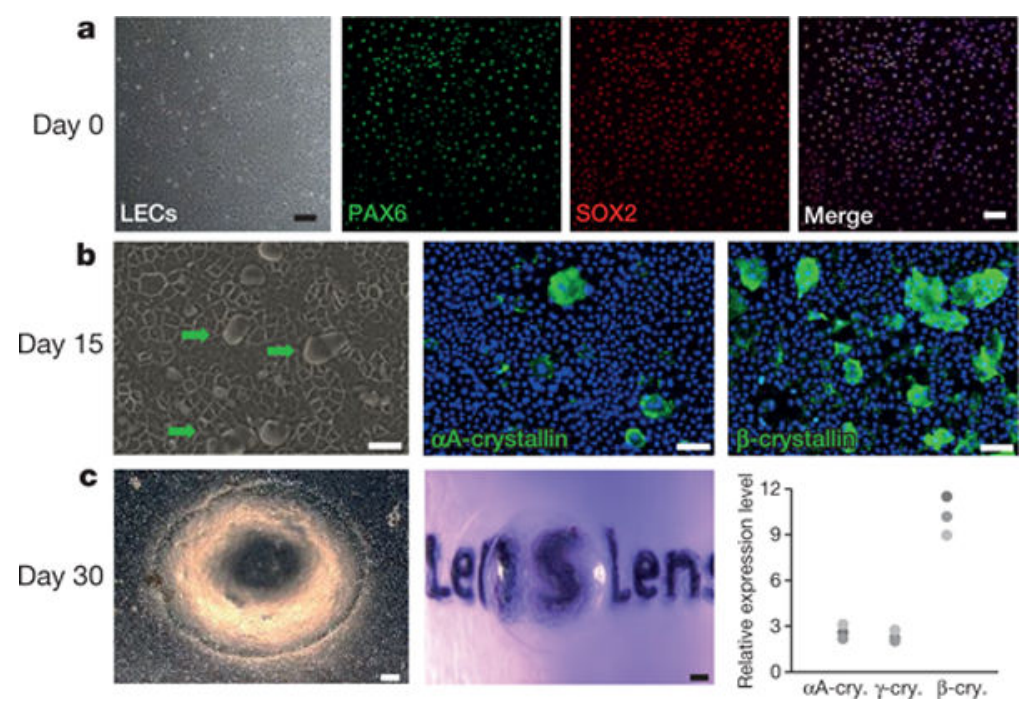

Figure 2. Characterization and differentiation of rabbit LECs

a, LECs were positive for PAX6 (green) and SOX2 (red). b, Lentoid formation (green arrows) with positive aA-crystallin and $\beta$-crystallin staining on day 15 of LEC

differentiation. c, Left panel, phase-contrast photograph of a lentoid body on day 30; middle panel, a lentoid body demonstrating magnifying properties; right panels, photograph from western blot analysis and quantification showing a dramatic increase in expression relative to pre-differentiation expression of mature lens fibre markers aA-crystallin $(2.6,3.1,2.2), \beta$ crystallin $(11.51,9.0,10.2)$ and $\gamma$-crystallin $(2.2,2.0,2.8)$ (numbers in parentheses represent fold change after differentiation). $n=3$ biological replicates. All scale bars, $100 \mu \mathrm{m}$. 
a

Minimally invasive cataract surgery

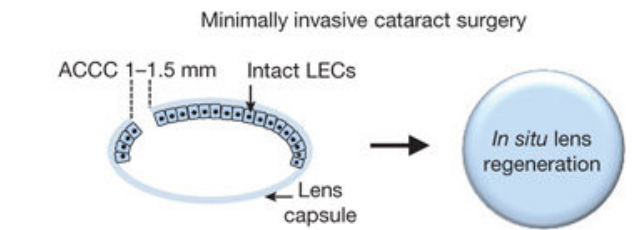

b
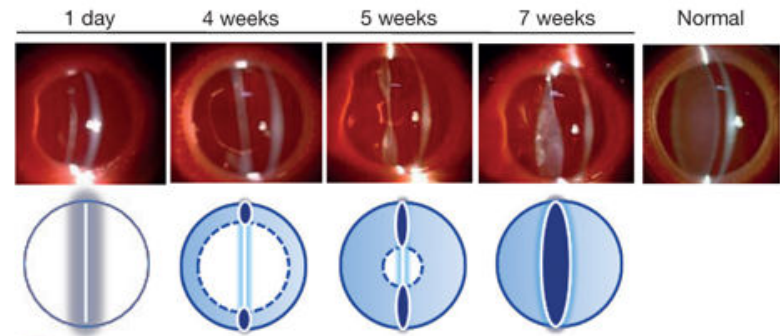

$\square$ Section view of the regenerated lens

$\square$ Coverage of the regenerated lens

c

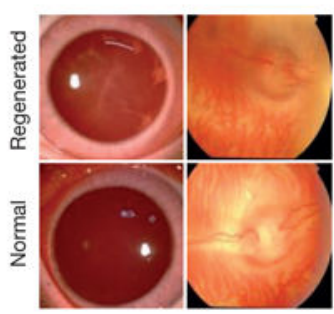

e

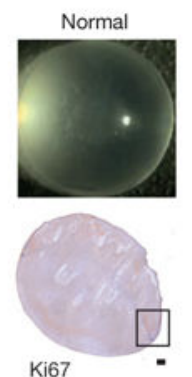

Ki67

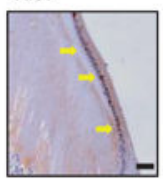

f
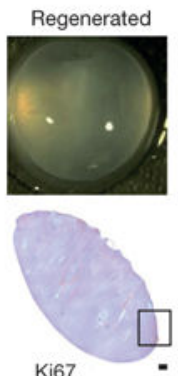

Ki67

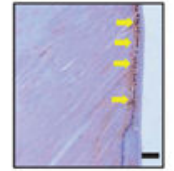

d

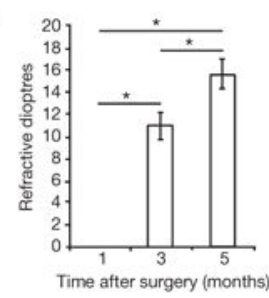

g
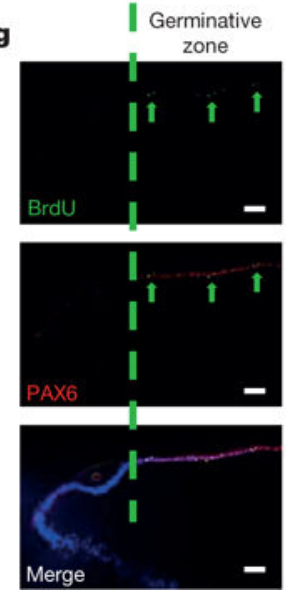

Figure 3. Lens regeneration in rabbits

a, New minimally invasive surgical method. The capsulorhexis size was decreased to 1.0$1.5 \mathrm{~mm}$ in diameter, resulting in a reduced wound area of $1.2 \mathrm{~mm}^{2}$, and moved to the periphery of the lens. $\mathbf{b}$, Slit-lamp microscopy showing the progress of lens regeneration after minimally invasive surgery in a rabbit eye. c, Fundus examination of rabbit eyes 7 weeks post-surgery demonstrated a clearly visible retina. Normal healthy lens shown for comparison. d, Measurements of refractive dioptres in rabbit eyes at different time points post-surgery (M, month; D, dioptres). Refractive dioptres of the eyes increased with time after surgery, demonstrating the functionality of the regenerated lenses (ANOVA, ${ }^{*} P<0.01$ ). The refractive power immediately after surgery was defined as zero, 1 month $=0.0$ dioptre, 3 months $=11.0 \pm 0.8$ dioptres and 5 months $=15.8 \pm 2.2$ dioptres, $n=6$ at each time point, data shown as means \pm s.d. e, $\mathbf{f}$, Ki67 staining in the germinative zone of normal rabbit lens 
(e) and regenerated rabbit lens 7 weeks post-surgery (f). Lower panels show higher magnification. g, PAX6 (red) and BrdU (green) staining at the germinative zone of regenerated rabbit lens 7 weeks post-surgery. Scale bars, $100 \mu \mathrm{m}$. 


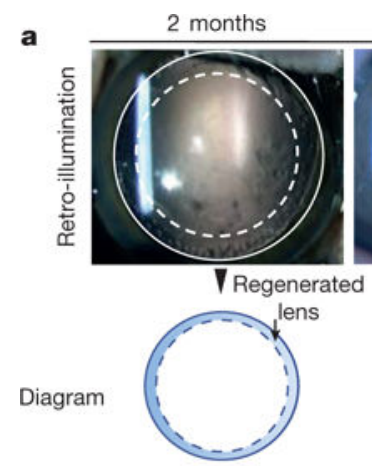

b
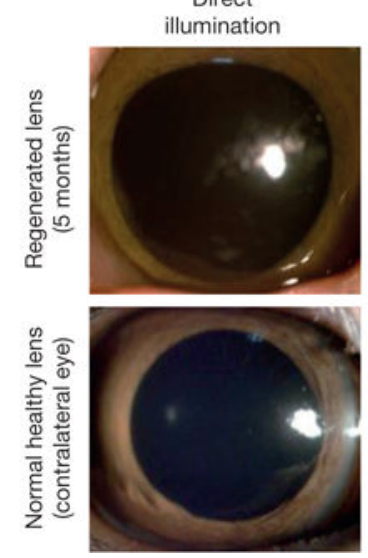

3 months

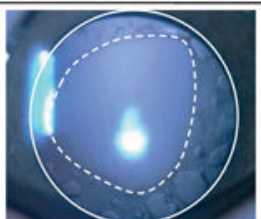

Regenerated

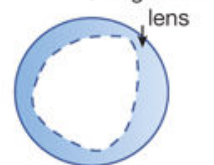

Sectional

view
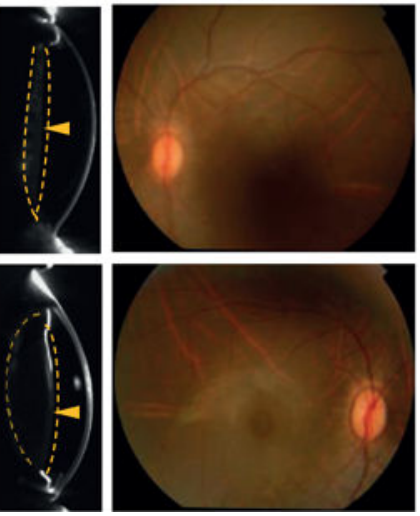

5 months

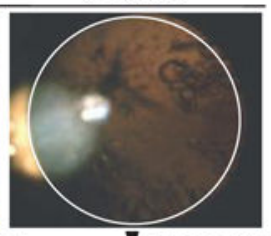

7 Regenerated

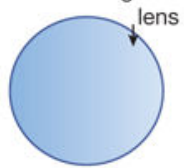

View of retina

Figure 4. Lens regeneration in macaque models after minimally invasive surgery

a, Slit-lamp microscopy showed regenerating lens tissue grew from the peripheral to the central lens in a circular symmetrical pattern 2-3 months after surgery, reaching the centre at 5 months post-surgery. Five months after surgery, direct illumination showed that the visual axis remained translucent. b, Pentacam cross-sectional scanning showed formation of a biconvex structure 5 months after surgery (yellow arrowheads). Direct illumination and fundus photography showed that the visual axis remained transparent and the retina was clearly visible $(n=6)$. 
a

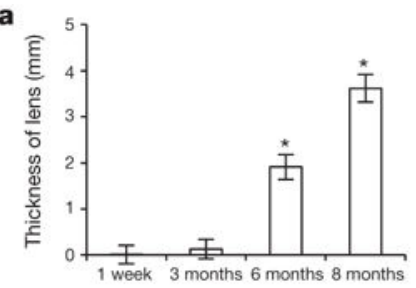

C
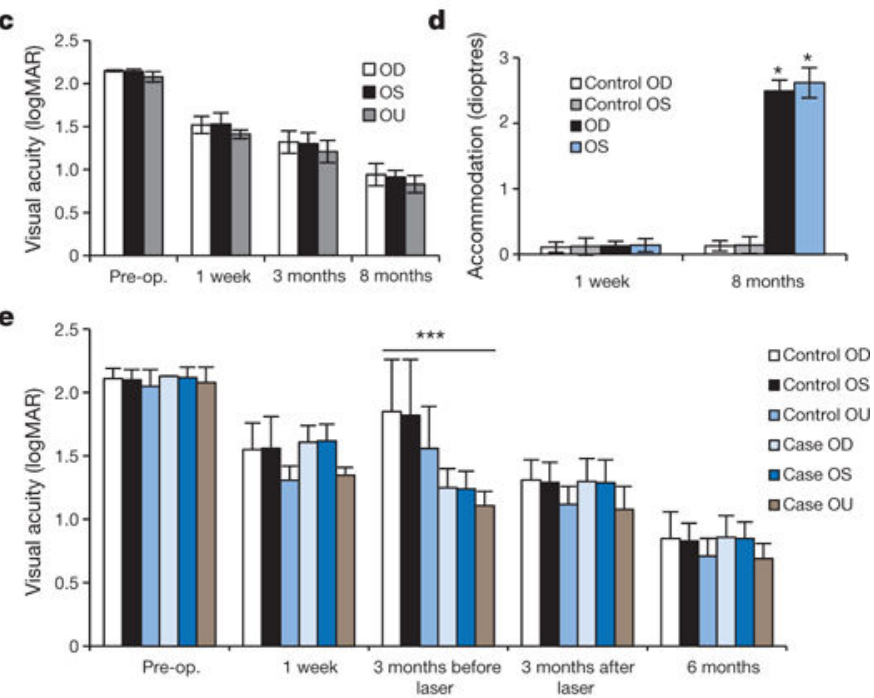

$\mathbf{f}$
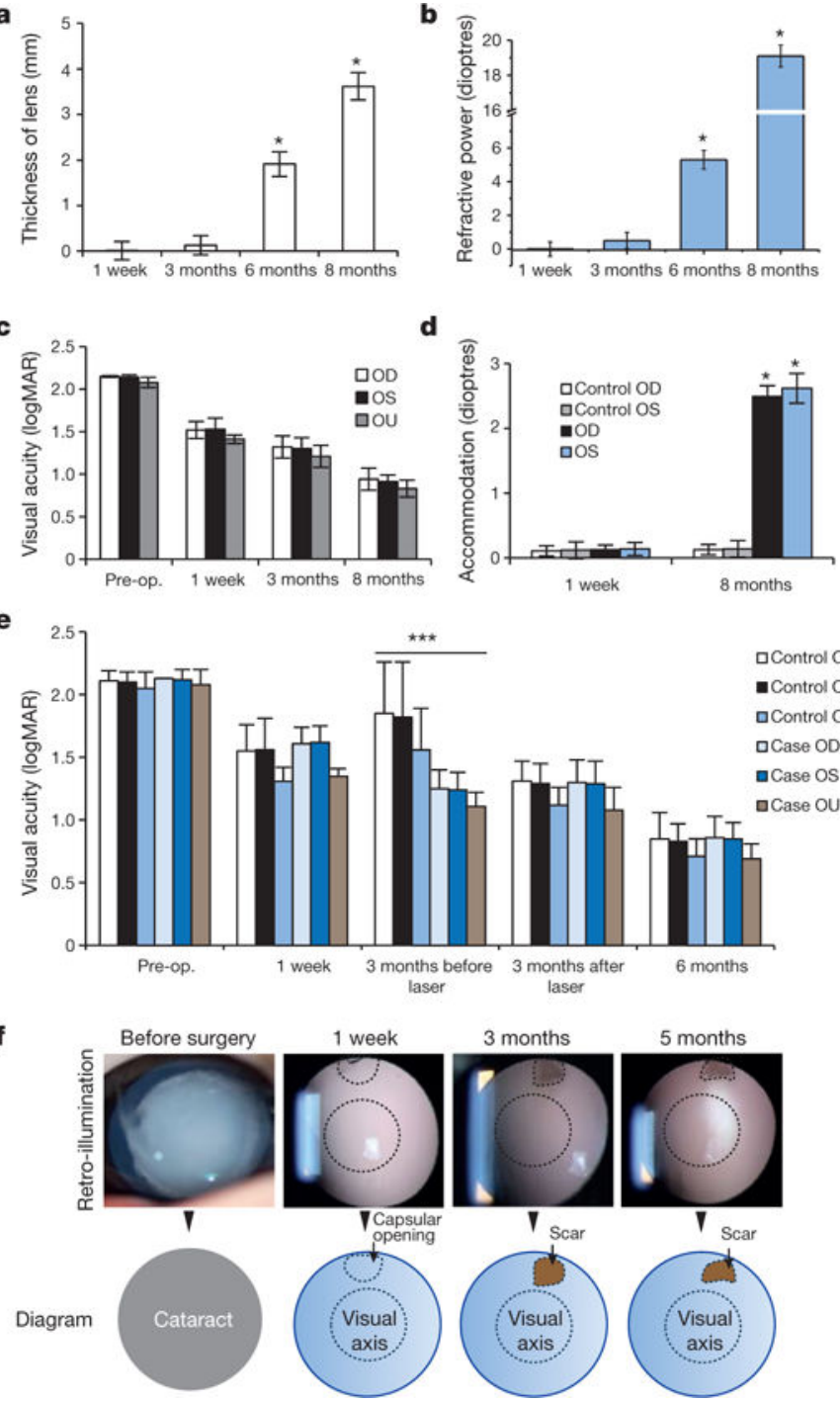

Figure 5. Functional characteristics of regenerated human lenses

a, Lens thickness increased significantly 6 and 8 months after surgery $(1.9 \pm 0.3$ and 3.7 $\pm 0.3 \mathrm{~mm}$, respectively, $\left.{ }^{*} P<0.01\right), n=24$. b. Lens refractive power increased significantly 6 and 8 months after surgery $\left(5.1 \pm 0.5\right.$ and $19.0 \pm 0.6$ dioptres, respectively, $\left.{ }^{*} P<0.01\right) . n=$ 24. c, Visual acuity improved after surgery. $\log M A R$, logarithm of the minimum angle of resolution. Pairwise analysis was performed to compare visual acuity before and after surgery (P < 0.05) OD (oculus dexter, right eye) $n=25$; OS (oculus sinister, left eye) $n=12$, OU (oculus uterque, both eyes) $n=12$. d, Accommodative power increased significantly from 1 week (control OD, control OS, OD and OS, all $0.1 \pm 0.1$ dioptres) to 8 months (control OD and control OS, $0.2 \pm 0.1$ dioptres; OD and OS, $2.5 \pm 0.2$ dioptres) postoperatively ( $* \mathrm{P}<0.001$ ). Control OD, $n=25$; control OS, $n=12$; OD, $n=12$; OS, $n=$ 12. e, Visual acuity was measured preoperatively and at 1 week, 3 months, and 6 months postoperatively. The majority of eyes in the control group underwent additional laser capsulotomy at 3 months after surgery, with visual acuity measured before and after the procedure. There was no significant difference in visual acuity between eyes that received 
minimally invasive surgery $(n=24)$ and those that received the current surgical technique $(n$ $=50$ ), except at 3 months before the control group underwent laser capsulotomy (t-test, $* * * P<0.001)$. Data are shown as mean \pm s.d. f, Visual axis transparency was achieved in nearly all cataractous infant eyes after minimally invasive surgery (95.8\%). The scar tissue of the wound on the anterior capsule was $<1.5 \mathrm{~mm}$ in diameter and located in the periphery, away from the visual axis. The scars were not visible unless the pupils were dilated. No disorganized tissue regeneration was observed. Compared with the current standard surgical method, the new surgical technique decreased VAO by $>20$-fold. 

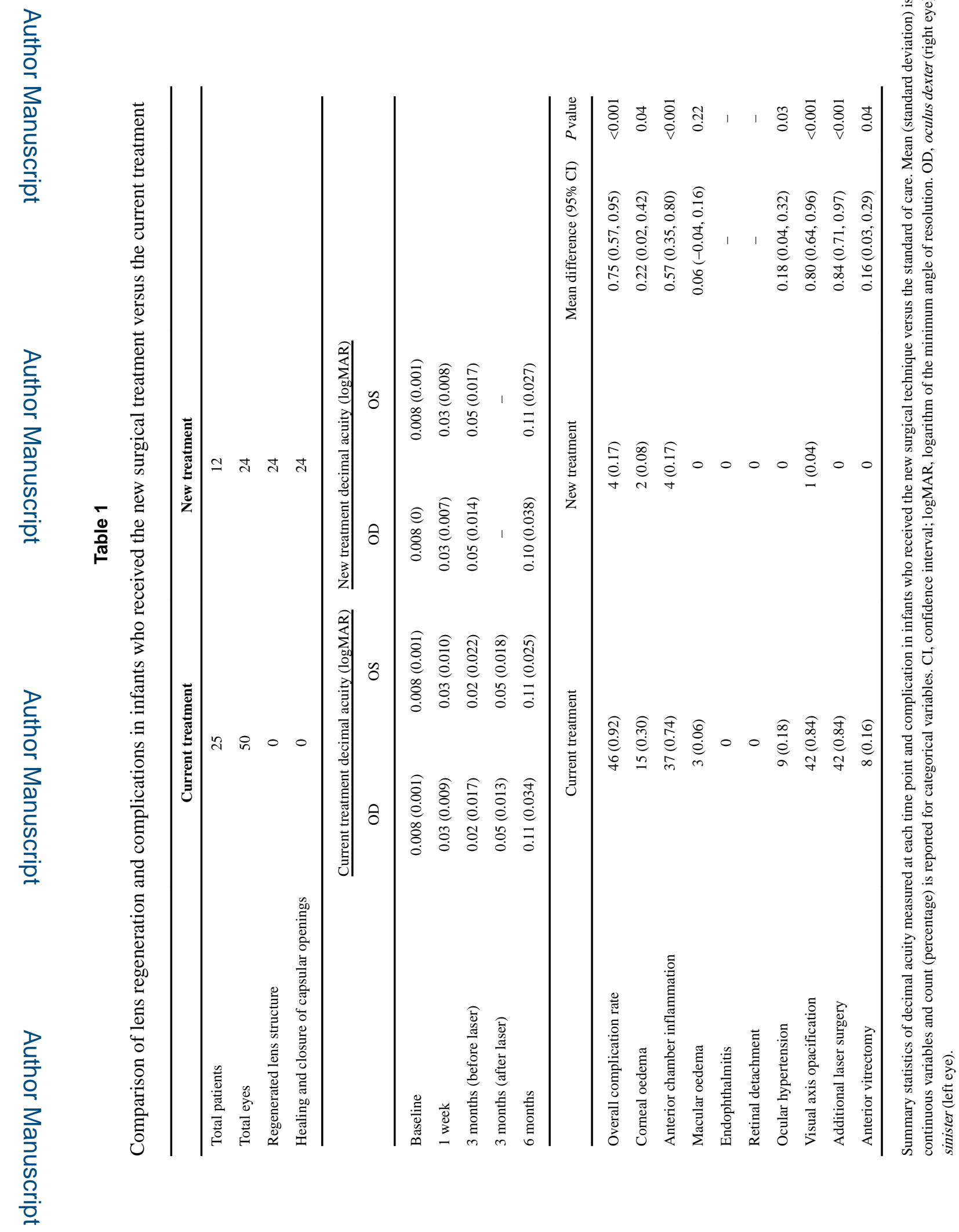

Nature. Author manuscript; available in PMC 2018 July 26. 